\title{
Structural and Functional Imaging with Carbon Nanotube AFM Probes
}

\section{Citation}

Hafner, Jason H., Chin Li Cheung, Adam T. Wooley, and Charles M. Lieber. 2001. Structural and functional imaging with carbon nanotube AFM probes. Progress in Biophysics \& Molecular Biology 77(1): 73-110.

\section{Published Version}

http://dx.doi.org/10.1016/S0079-6107(01)00011-6

\section{Permanent link}

http://nrs.harvard.edu/urn-3:HUL.InstRepos:2757769

\section{Terms of Use}

This article was downloaded from Harvard University's DASH repository, and is made available under the terms and conditions applicable to Other Posted Material, as set forth at http:// nrs.harvard.edu/urn-3:HUL.InstRepos:dash.current.terms-of-use\#LAA

\section{Share Your Story}

The Harvard community has made this article openly available.

Please share how this access benefits you. Submit a story.

Accessibility 
Published in Progress in Biophysics \& Molecular Biology 77 (2001), pp. 73-110; doi:10.1016/S0079-6107(01)00011-6

Copyright (C 2001 Elsevier Science Ltd. Used by permission. Published online July 20, 2001.

http://www.sciencedirect.com/science/journal/00796107

\title{
Review
}

\section{Structural and functional imaging with carbon nanotube AFM probes}

\author{
J. H. Hafner, C. -L. Cheung, A. T. Woolley, and C. M. Lieber* \\ Department of Chemistry and Chemical Biology, Harvard University, Cambridge, MA 02138, USA \\ * Corresponding author_tel 617 495-9833; fax: 617 496-5442; email cml@cmliris.harvard.edu
}

\begin{abstract}
Atomic force microscopy (AFM) has great potential as a tool for structural biology, a field in which there is increasing demand to characterize larger and more complex biomolecular systems. However, the poorly characterized silicon and silicon nitride probe tips currently employed in AFM limit its biological applications. Carbon nanotubes represent ideal AFM tip materials due to their small diameter, high aspect ratio, large Young's modulus, mechanical robustness, well-defined structure, and unique chemical properties. Nanotube probes were first fabricated by manual assembly, but more recent methods based on chemical vapor deposition provide higher resolution probes and are geared towards mass production, including recent developments that enable quantitative preparation of individual single-walled carbon nanotube tips [J. Phys. Chem. B 105 (2001) 743]. The high-resolution imaging capabilities of these nanotube AFM probes have been demonstrated on gold nanoparticles and well-characterized biomolecules such as IgG and GroES. Using the nanotube probes, new biological structures have been investigated in the areas of amyloid-beta protein aggregation and chromatin remodeling, and new biotechnologies have been developed such as AFM-based haplotyping. In addition to measuring topography, chemically functionalized AFM probes can measure the spatial arrangement of chemical functional groups in a sample. However, standard silicon and silicon nitride tips, once functionalized, do not yield sufficient resolution to allow combined structural and functional imaging of biomolecules. The unique end-group chemistry of carbon nanotubes, which can be arbitrarily modified by established chemical methods, has been exploited for chemical force microscopy, allowing single-molecule measurements with well-defined functionalized tips.
\end{abstract}

Keywords: atomic force microscopy, carbon nanotube, chemical force microscopy, single molecule spectroscopy, structural biology 
Contents

1. Introduction 74

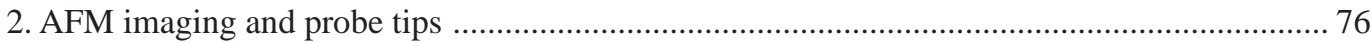

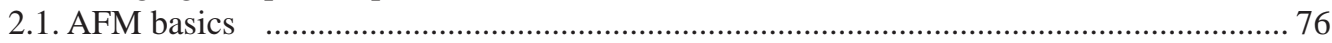

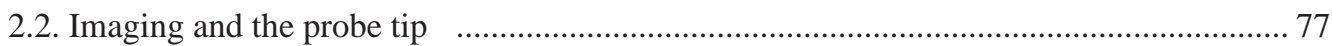

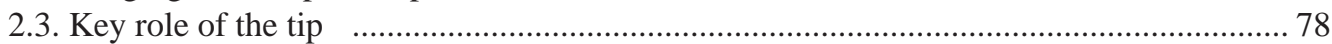

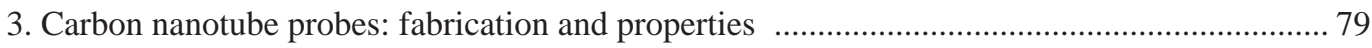

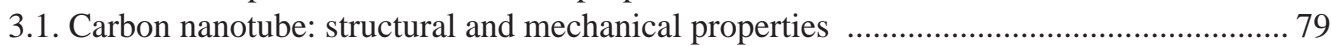

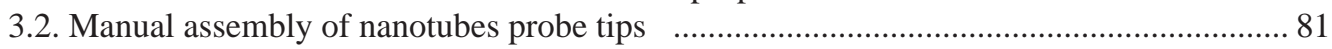

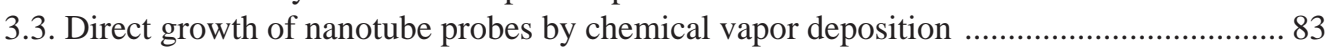

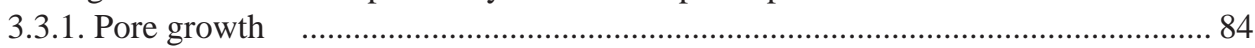

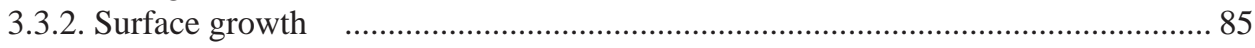

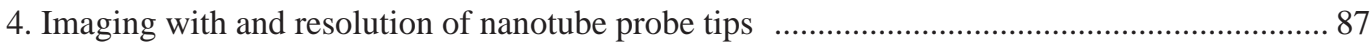

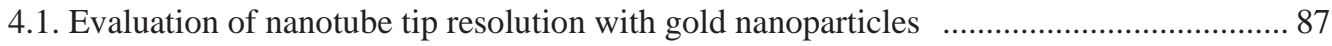

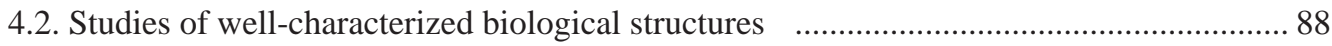

5. Structural biology and other applications of nanotube tips ......................................................... 91

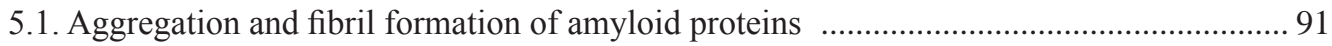

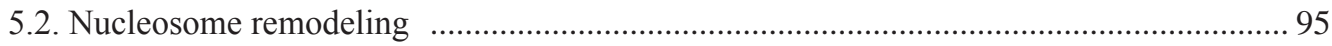

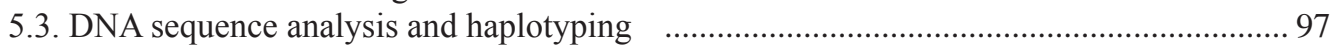

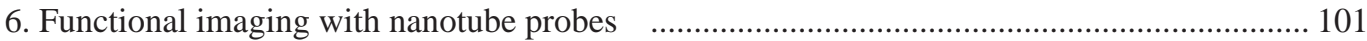

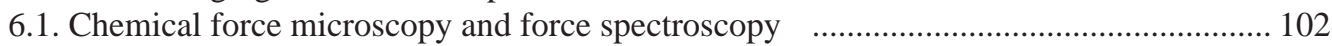

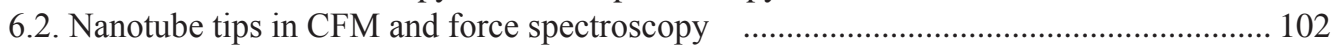

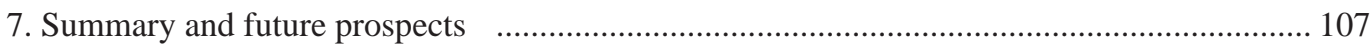

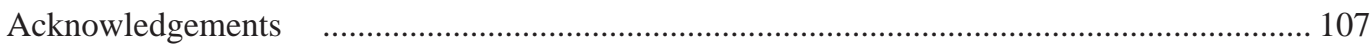

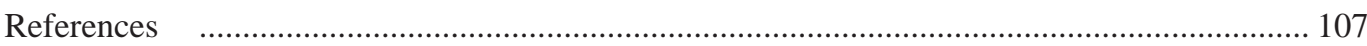

\section{Introduction}

Elucidating the three dimensional structures of proteins, nucleic acids and assemblies of these macromolecules is critical to developing a molecular level understanding of biological function. X-ray diffraction, electron diffraction, and nuclear magnetic resonance (NMR) are well-established and powerful tools for the analysis of macromolecular structure with atomic precision, which with advances in technology, have continued to push the limits of the size and complexity of systems that can be structurally illuminated (Beauchamp and Isaacs, 1999; Chiu et al., 1999; Glaeser, 1999; Glusker, 1993; Henderson, 1995; Siegal et al., 1999; Stowell et al., 1998; Wuthrich, 1995). As one looks to the future, the needs for and impact of macromolecular structure analysis are expected to expand significantly in several areas, including: (1) increased throughput of structural analysis of gene products discovered by 
large scale genome sequencing (Kim, 1998); (2) routine characterization of large multimeric structures, such as protein assemblies and protein-nucleic acid complexes involved in signaling and regulation; and (3) understanding dynamic features of protein-protein and protein-nucleic acid assemblies. The standard structural tools can impact each of these areas but also have limitations. For example, difficulties in crystallization of large multimeric signaling and regulatory complexes may limit the general use of X-ray and electron diffraction methods for these critical problems, while solution NMR studies of macromolecular dynamics (and structure) have size restrictions that limit the systems that can be studied (Wuthrich, 2000).

It thus seems clear that future progress in elucidating the complex machinery of the cell will depend in part on additional and perhaps revolutionary developments in structural biology tools. In this regard, two complementary techniques are receiving increased attention: (1) cryogenic transmission electron microscopy (cryoEM) and (2) atomic force microscopy (AFM). CryoEM allows the direct visualization of biological structures, and can produce high-resolution images of isolated proteins with resolution of 10-25 A (Grigorieff, 1998; Kuhlbrandt and Williams, 1999). Because contrast is low in biological EM, the high resolution achieved by cryoEM requires computer averaging and processing of thousands of images. The ability to determine structure from single macromolecule images represents a distinct advantage for the large multimeric systems discussed above. AFM allows direct visualization of biological structures like cryoEM, and moreover, can probe structural dynamics in vitro since structural imaging can be carried out in solution (for reviews, see Shao et al., 1996; Engel et al., 1997; Fritz et al., 1995; Bustamante et al., 1997). This latter feature of AFM represents a distinct advantage compared to cryoEM.

In previous work, AFM has been used to probe the structures of a number of protein and nucleic acid systems. Key results obtained from past studies include (1) resolution of the packing and often subunit arrangement of membrane proteins (Muller and Muller; Scheuring et al., 1999; Shao et al., 1996), (2) determination of the overall structures of protein-DNA complexes (Bustamante and Rivetti, 1996), and (3) elucidation of dynamic processes (Kasas et al., 1997; Guthold et al., 1999). In addition, in chemical force microscopy (CFM), it has been shown that the AFM tip can be modified to present well-defined chemical or biological functionality, which provides specific chemical contrast as well as topography (Frisbie et al., 1994; Fujihira and Ohzono, 1999; Green et al., 1995; Noy et al., 1997b; Vezenov et al., 1997). These capabilities of AFM suggest a great potential to impact many areas of structural biology, although to date the impact on major structural and dynamic problems has been much less than that achieved by electron microscopy as well as X-ray diffraction and NMR.

When we ask how the significant potential of AFM might be better exploited in structural biology, one needs to look no further than the structure of the tip used for imaging. Since an AFM image is a convolution of the structure of the macromolecule or macromolecular assembly under investigation and the tip structure, a well-characterized tip is essential for accurately interpreting an image, and moreover, the size of the tip will define the resolution of the image. The situation is somewhat analogous to the need for well-collimated and monoenergetic electron beams in cryoEM and well-defined X-ray sources in X-ray diffraction. In the following review, we will focus on recent and significant progress in this area of AFM technology and new and potential science enabled by the advances in AFM probes. The structure of the review will be as follows. First, we will discuss briefly the basics of AFM and imaging biological structures with an emphasis on highlighting key issues for probe tips. Second, we will describe the fabrication and characterization of a new generation of tips based on carbon nanotubes. Third, we will examine the reproducibility and resolution of these probes in imaging relatively well- 
defined inorganic and biological structures. Fourth, we will describe studies that are beginning to exploit the unique features of these new probes for addressing new structural biology and other problems. Fifth, we introduce the concept of CFM and describe the unique potential of carbon nanotube tips for functional imaging at the nanometer scale. Lastly, we briefly summarize and speculate on prospects of carbon nanotubes probes in the future.

\section{AFM imaging and probe tips}

\subsection{AFM basics}

To understand the strengths and weaknesses of AFM, we first consider the principles of operation. A typical AFM (Figure 1) consists of an integrated cantilever-tip assembly, a detector to measure can-

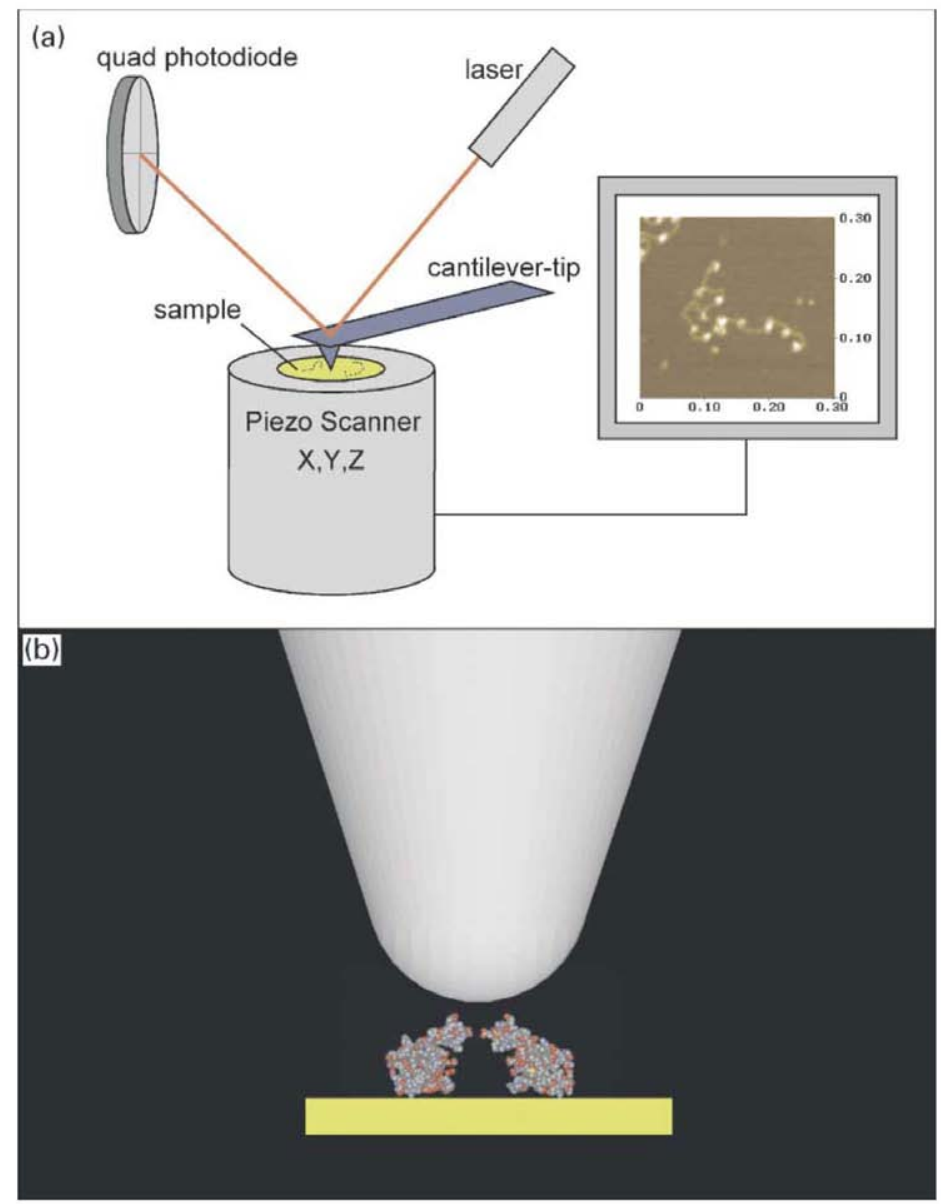

Figure 1. A schematic of the atomic force microscope: (a) The cantilever-tip assembly, piezo tube scanner, laser deflection system, and computer are depicted. (b) Comparison of a $5 \mathrm{~nm}$ radius of curvature cone-shaped tip to a cross section of an individual GroES molecule, which highlights the limitations of such tips in high-resolution imaging. 
tilever displacement and feedback electronics to maintain a constant imaging parameter, such as force or tip-sample separation. The sample, which is supported on a solid substrate surface, can be scanned in three-dimensions $(X, Y, Z)$ with angstrom precision using a three-axis piezoelectric translation stage. The tip position is monitored with an optical beam deflection system. Tip deflections on the order of angstroms, which correspond to forces of approximately $10 \mathrm{pN}$, can be reliably measured using standard cantilevers. Recently, higher resonance frequency cantilevers have been developed (Viani and Viani). These cantilevers extend both the potential imaging speed and force resolution by approximately one order of magnitude, and thus should be attractive for biological studies.

There are two basic modes with which structural images can be acquired. In contact mode, the tip remains in contact with the sample/substrate, while scanning in a $(X, Y)$ raster pattern. Typically, the $Z$ position is adjusted during scanning to maintain constant cantilever deflection (i.e., constant force) at a set point value. The resulting $Z$ data represent a topographic image of the surface. Contact mode imaging is best for relatively flat and hard samples since the scanning tip applies large lateral forces. Attempts to image isolated biological structures usually result in poor resolution and sample movement or damage. If the forces are very carefully controlled, however, contact mode can provide high-resolution images of 2-D protein arrays (Muller and Muller). Alternatively, images can be acquired using intermittent contact or tapping mode. In this mode, the cantilever-tip assembly is oscillated near resonance and when brought to the surface intermittently contacts - taps - the sample. To image in tapping mode, the $Z$ position is adjusted to maintain the oscillation amplitude at a constant set point during the $(X-Y)$ scan. The significant advantage of tapping mode is that there are essentially no lateral forces applied to a sample during imaging, and thus it is the technique of preference for probing isolated macromolecules, macromolecular assemblies and the dynamics of these systems.

\subsection{Imaging and the probe tip}

The structural resolution of AFM will depend strongly on the nature of the probe tip, and thus it is instructive to reference state-of-the-art microfabricated tips to known protein structures. For example, Figure 1b compares the relative sizes (in cross section) of a $5 \mathrm{~nm}$ radius of curvature silicon tip and the ca. $8 \mathrm{~nm}$ diameter heptameric protein GroES. This comparison clearly suggests that conventional tips will not provide significant structural detail.

Experimental studies of isolated proteins and nucleic acids, and two-dimensional protein arrays indicate that the situation is more complex. AFM investigations of isolated DNA have shown several features, including kinks (Han et al., 1997) and supercoiled structures (Lyubchenko and Schlyakhtenko, 1997). Resolution on the order of 1-2 nm has been reported in these studies, although the $3.4 \mathrm{~nm}$ helical pitch of DNA was not resolved. Earlier studies of isolated DNA (Hansma et al., 1995) and condensed DNA arrays (Mou et al., 1995) did show the DNA helical pitch, although this structure has not been observed reproducibly in subsequent studies. Moreover, it should be noted that the best reported lateral resolution was achieved using tips having average radii of curvature on the order of $50 \mathrm{~nm}$. AFM imaging of other isolated biological structures have produced various levels of resolution. Membrane bound E. coli F1F0 ATP synthase revealed a ring-like structure with an off center mass at resolution of ca. $3 \mathrm{~nm}$ (Singh et al., 1996). Several studies of immunoglobulin G (IgG) failed to resolve the characteristic $15 \mathrm{~nm}$ Y-shape of this macromolecule (Bergkvist et al., 1998), while others imaged the Y-shape 
as a heart-like structure (Fritz et al., 1997). However, the Y-shape was apparent at cryogenic temperatures (Zhang et al., 1996). These widely varying results illustrate the sensitivity of high-resolution biological AFM to imaging conditions and tip quality. Comparison of these different studies shows that the highest resolutions were found in low-height structures (i.e., DNA and the membrane bound protein), while isolated proteins (e.g., IgG) showed significantly lower resolution with conventional tips. Hence, these results suggest that roughness or asperities at the tip end might be responsible for the high-resolution images of DNA and membrane structures, although such asperities have not been independently characterized.

In addition, very high resolution has been observed in AFM studies of densely packed two-dimensional arrays of proteins (Muller and Muller). For example, 5-6 A lateral resolution has been reported for images of bacteriorhodopsin (Muller et al., 1999b), even though the silicon nitride tips used for imaging have radii of 20-60 $\mathrm{nm}$. To explain this apparent contradiction, it has been hypothesized that the image contrast is due to short-range repulsive forces on a small nano-protrusion at the tip end (Muller et al., 1999b). This explanation is similar to that used to explain high-resolution images obtained on isolated low-height molecules, such as DNA. Another possibility is that the subnanometer resolution corresponds to Fourier components from the periodic protein lattice. This latter idea is supported by the fact that the highest resolution (Muller et al., 1999b) has been achieved for proteins in a crystalline 2D array, while approximately two-fold lower resolution has been observed in less well-ordered arrays (Mou et al., 1996); that is, the reduced positional and orientational order would produce lower resolution in the Fourier model. Lastly, the observation of missing-molecule defects may be consistent with both models, since the highly nonlinear tip-sample interaction can still produce an apparent defect in the Fourier model.

\subsection{Key role of the tip}

The studies reviewed above show that microfabricated tips can provide resolution much higher than expected based on the smooth end radii of curvature of tips. While observed high resolution can be reasonably attributed to asperities at the tip ends, this poses several important issues for the general use of AFM in high-resolution structural biology. First, the presence of these asperities has not been characterized independently by techniques such as transmission electron microscopy. Second, the observation of high resolution is highly dependent on obtaining a "good" tip and cannot be predicted a priori. Moreover, current tips have not been able to image large, isolated protein structures, which form the basis of much of the cell signaling and regulatory machinery, with high resolution. We believe that these uncertainties in the detailed nature of the probe tip must be addressed in order to move AFM to the level of other structural biology techniques for addressing new scientific problems.

Let us take a step back and examine microfabricated tips and the requirements for an ideal tip. Integrated AFM cantilever-tip assemblies are fabricated from silicon ( $\mathrm{Si}$ ) or silicon nitride $\left(\mathrm{Si}_{3} \mathrm{~N}_{4}\right)$ (Albrecht et al., 1989). The tips on these assemblies are pyramidal in shape, have cone angles of $20-30^{\circ}$ and radii of curvature of $5-10 \mathrm{~nm}(\mathrm{Si})$ or $20-60 \mathrm{~nm}\left(\mathrm{Si}_{3} \mathrm{~N}_{4}\right)$. Several techniques have been developed to improve these geometrical factors. $\mathrm{Si}_{3} \mathrm{~N}_{4}$ tips can be oxide sharpened to improve their aspect ratio and reduce the tip radii to as low as $5 \mathrm{~nm}$. Silicon tips can be machined with focused ion beam (FIB) instruments to improve the aspect ratio, and sharper Si tips are now available with a high yield of radii less 
than $5 \mathrm{~nm}$. Despite these technological advances, there remain important limitations. For example, the variation in tip-to-tip properties can be quite large, and will always be difficult to control at the scale relevant to high-resolution structural imaging. In addition and critical to reproducibility, tips wear during scanning (Hansma et al., 1995), making it quite difficult to account quantitatively for tip contributions to image broadening. The problem of tip wear will only increase for sharper tips due to increased pressure at the tip-sample interface.

Consider the ideal AFM tip. It should have a high aspect ratio with $0^{\circ}$ cone angle, have a radius as small as possible with well-defined and reproducible molecular structure, and be mechanically and chemically robust such that its structure is not altered while imaging in air or fluid environments. Carbon nanotubes are the only known materials that can satisfy all of these critical criteria, and thus have the potential to create ideal probes for AFM imaging. Below we review the key properties of carbon nanotubes and methods to create tips with this unique class of material.

\section{Carbon nanotube probes: fabrication and properties}

\subsection{Carbon nanotube: structural and mechanical properties}

Carbon nanotubes consist of a honeycomb $\mathrm{sp}^{2}$ hybridized carbon network (termed a graphene sheet) that is rolled up into a seamless cylinder (Figure 2a), which can be microns in length. There are two basic structural classes of carbon nanotubes: single-walled nanotubes (SWNTs) and multiwalled nanotubes (MWNTs). SWNTs consist of a single seamless cylinder (Figure 2a) with radii ranging from 0.35 to $2.5 \mathrm{~nm}$, while MWNTs consist of multiple concentric graphene cylinders with radii ranging from 3 to $50 \mathrm{~nm}$. Transmission electron microscopy (TEM) images of an individual MWNT and SWNT are displayed in Figs. 2b and c, respectively. These TEM images show clearly the tubular structure of the nanotubes, and in the case of the SWNT (Figure 2c) highlight the extremely small diameter and high aspect ratio, which makes this material uniquely suited as a high-resolution probe tip.

Nanotubes have exceptional mechanical properties. The characteristics relevant to the use of nanotubes as AFM tips are the stiffness or Young's modulus and the ability to buckle elastically under large loads. Recent calculations of SWNT and MWNT Young's moduli suggest that both classes of nanotube have moduli on the order of $1 \mathrm{TPa}(\mathrm{Lu}, 1997)$. Experimental measurements of MWNTs Young's moduli made by recording thermal vibration amplitudes in a TEM (Treacy et al., 1996) and by direct bending with AFM (Wong et al., 1997) revealed Young's moduli of 1.8 and $1.3 \mathrm{TPa}$, respectively. More recent studies of individual 1.0-1.5 nm diameter SWNTs found a similar Young's modulus, $1.25 \mathrm{TPa}$ (Krishnan et al., 1999). Hence, the theoretical and experimental results are in good agreement, and show that nanotubes are stiffer than any other known material. The extremely high Young's modulus of nanotubes is critical to the creation of high aspect ratio, sub-nanometer radius tips with high resolution-if the modulus were significantly smaller, then the amplitude of thermal vibrations would degrade the resolution of tips.

Carbon nanotubes buckle elastically under large loads unlike all conventional materials, which either fracture or plastically deform. MWNTs and SWNTs have been observed in the buckled state by TEM (Iijima et al., 1996), and appear to buckle in a manner strikingly similar to macroscopic tubes made of 


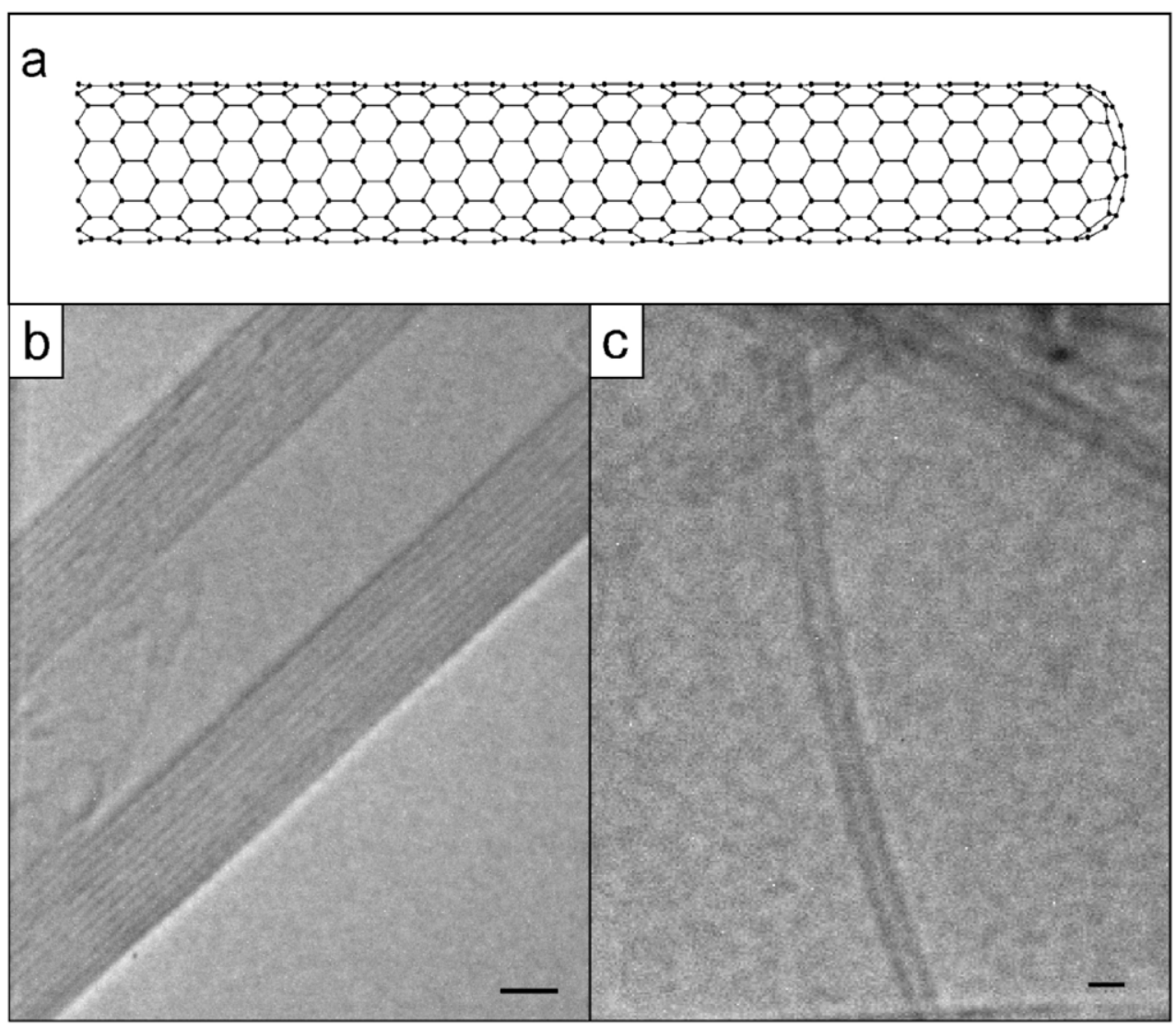

Figure 2. The structure of carbon nanotubes: (a) Fragment of a stick model of a single-walled carbon nanotube. Each vertex in the honeycomb mesh corresponds to a sp²-bonded carbon atom. Transmission electron micrographs show the cross-sectional structures of (b) a multi-walled nanotube and (c) a single-walled nanotube.

elastic materials. The first direct experimental evidence that carbon nanotube buckling is elastic came from the use of nanotubes as AFM tips (Dai et al., 1996). The buckling force of the nanotube tip could be measured from the deflection of the AFM cantilever as the nanotube tip-surface separation was reduced. The nanotube would then return to its original configuration when the tip was removed from the surface. A more direct measurement of elastic nanotube buckling was achieved by AFM measurements of force versus displacement of nanotubes pinned at one end on a surface (Wong et al., 1997). Both types of experiments demonstrated that nanotubes can be bent close to $90^{\circ}$ many times without observable damage, and thus should be highly robust probes for AFM imaging. 


\subsection{Manual assembly of nanotube probe tips}

The first carbon nanotube AFM tips were produced by manually attaching MWNTs to the pyramids of conventional tips (Dai et al., 1996). In this process, micromanipulators are used to control independently the positions of a commercial cantilever-tip assembly and nanotubes, while viewing in an optical microscope. The nanotubes and tip are readily seen under dark-field illumination at 500-1000× magnification. A thin layer of adhesive is applied to the pyramidal silicon tip by touching the surface of adhesive tape that supports the nanotube material. The pyramidal tip is then used to pull an MWNT or bundle of MWNTs from the tape surface. Micromanipulators allow the fabrication of nanotube tips that are well aligned for AFM imaging; that is, they are parallel to the tip axis and therefore perpendicular to the sample surface. However, the attached nanotube tips are typically too long to permit highresolution imaging. The vibration amplitude at the end of the tip, $X_{\text {tip }}$, can be readily estimated from $X_{\text {tip }}=\left[\left(4 k_{\mathrm{B}} T L^{3}\right) /\left(3 \pi E r^{4}\right)\right]^{1 / 2}$, where $k_{\mathrm{B}}$ is the Boltzmann's constant, $T$ is the temperature in Kelvin, $L$ is the length, $E$ is the Young's modulus and $r$ is the radius of the nanotube tip. This estimate of the vibration amplitude, which assumes $1 / 2 k_{\mathrm{B}} T$ thermal energy in the nanotube tip, works well for both individual and bundle nanotube tips since the Young's modulus of a bundle of nanotubes is essentially the same as an individual nanotube (Lu, 1997). The length of a nanotube tip can be decreased by electrical etching on a conductive surface (Dai et al., 1996; Wong et al., 1998a) to reduce the amplitude of vibration to a level where it does not affect resolution.

Mechanically assembled MWNT tips have demonstrated several important features. First, the high aspect ratio of the tips enables more accurate images of structures with steep sidewalls such as silicon trenches (Dai et al., 1996). Second, the tip-sample adhesion is greatly reduced due to the small size and cylindrical geometry of the nanotube (Dai et al., 1996; Wong et al., 1998a). Reduced adhesion allows imaging with lower cantilever energies and less potential sample damage. Third, they have demonstrated clearly the elastic buckling property of nanotubes. For example, as an oscillating nanotube tip is brought into contact with a sample, the cantilever oscillation amplitude is damped strongly but then partially recovers when the nanotube buckles as shown in Figure 3. The deflection at the buckling point corresponds to the Euler buckling force, $F_{\mathrm{B}}=\pi^{2} E I / L^{2}$, where $E$ is the Young's modulus, $I=\pi r^{4} / 4$ is the moment of inertia, $L$ is the nanotube tip length, and $r$ is the nanotube radius. This elastic buckling behavior has tremendous consequences for nanotube tip robustness, and arises from the $\mathrm{sp}^{2}$ bonded cylindrical structure of carbon nanotubes. In deflecting carbon nanotubes and carbide nanorods with an AFM tip, it was found that nanotubes elastically buckle while nanorods fracture (Wong et al., 1997).

MWNT tips were found to provide only a modest improvement in resolution compared with standard silicon tips when imaging isolated amyloid fibrils (Wong et al., 1998a). The clear route to higher resolution is to use SWNTs, since they typically have $0.5-2 \mathrm{~nm}$ radii. SWNT material produced by laser vaporization of a graphite target doped with metal catalyst consists of high-purity SWNTs (Thess et al., 1996). Unfortunately, the SWNTs produced in this way are aligned in bundles approximately $10 \mathrm{~nm}$ wide containing up to hundreds of nanotubes each. These bundles can be readily attached to silicon AFM tips (Wong; Wong and Wong), and their lengths adjusted by the electrical etching procedure described above for optimal imaging. Significantly, this etching process was found to occasionally produce very high-resolution tips that likely resulted from the exposure of only a small number of SWNTs 

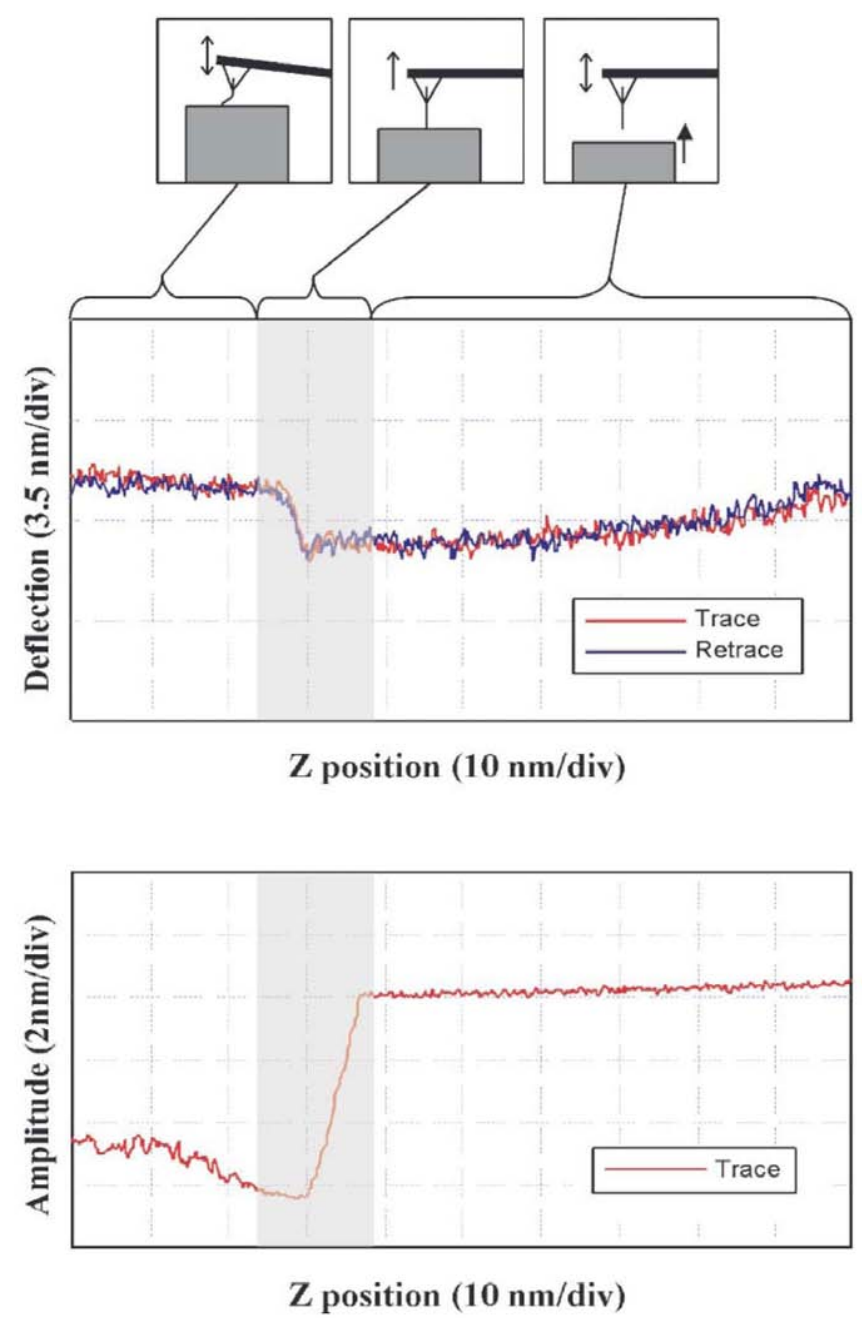

Figure 3. Nanotube tip buckling: The oscillation amplitude and deflection of a nanotube tip are plotted as a function of tip-sample separation. To the right of the shaded region, the tip and sample are out of contact, so the amplitude is constant and the deflection is zero. In the shaded region, the tip taps the sample resulting in reduced amplitude and tip deflection. To the left of the shaded region, the nanotube has buckled, which results in a leveling off of the cantilever deflection and an increase of the oscillation amplitude.

at the apex (Wong and Wong). It was not possible in these studies to prepare reproducibly individual SWNT tips for imaging.

The manual assembly method of producing nanotube tips is conceptually straightforward but also has several important limitations. First, this assembly procedure inherently leads to the selection of thick bundles of nanotubes since these are easiest to observe in the optical microscope. Recently, mechanical assembly of nanotube tips was also performed inside a scanning electron microscope (SEM) (Nishijima et al., 1999). The use of an SEM still limits assembly to nanotube bundles or individual tubes with diameters greater than $5-10 \mathrm{~nm}$, and moreover, increases greatly the time required to make 
one tip. Second, well-defined and reproducible tip etching procedures designed to expose individual SWNTs at the tip apex do not exist. Third, a relatively long time is required to attach each nanotube to an existing cantilever. This not only inhibits carrying out the research needed to develop these tips but also precludes mass production required for general usage. Nevertheless, nanotube tips are now being produced by this method and sold commercially ( http://www.piezomax.com ), although each tip is quite expensive.

\subsection{Direct growth of nanotube probes by chemical vapor deposition}

All of the problems associated with manual assembly potentially can be solved by directly growing nanotubes onto AFM tips by metal-catalyzed chemical vapor deposition (CVD). The key features of the nanotube CVD process are illustrated in Figure 4. In the CVD synthesis of carbon nanotubes, metal catalyst particles are heated in a gas mixture containing hydrocarbon or $\mathrm{CO}$. The gas molecules dissociate on the metal surface and carbon is adsorbed into the catalyst particle. When this carbon precipitates, it nucleates a nanotube of similar diameter to the catalyst particle. Hence, CVD allows control over nanotube size and structure including the production of SWNTs (Hafner et al., 1998), with radii as low as $3.5 \AA$ (Nikolaev et al., 1999).

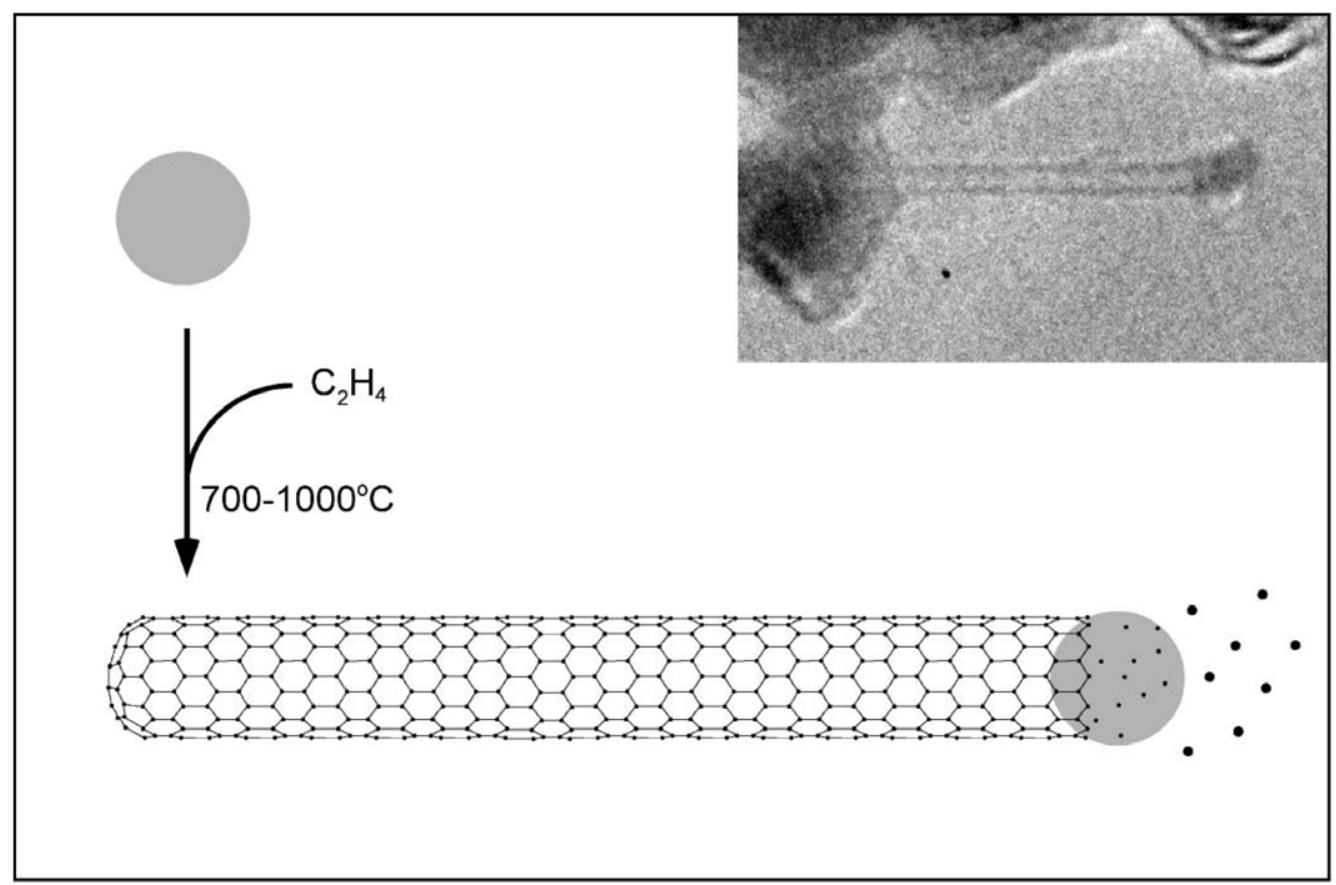

Figure 4. Nanotube production by CVD: Nanometer-scale catalyst particles are heated in the presence of a hydrocarbon gas or carbon monoxide. The reactant molecules dissociate on the surface of the catalyst particle, carbon is adsorbed into the particle, and precipitates from the particle as a nanotube. In the upper right is a TEM image of a ca. $2 \mathrm{~nm}$ diameter single-walled nanotube with the catalyst nanoparticle attached as shown schematically in the diagram. 


\subsubsection{Pore growth}

The central issues in the growth of nanotube AFM tips by CVD are (1) how to align the nanotubes at the tip such that they are well positioned for imaging and (2) how to ensure there is only one nanotube or nanotube bundle at the tip apex. In previous studies of bulk nanotube production, it was found that nanotubes grew perpendicular to a porous surface containing embedded catalyst (Li et al., 1996). We exploited this approach in the first demonstration of CVD nanotube tips (Hafner et al., 1999a) as illustrated in Figure 5. A flattened area of approximately $1-5 \mu \mathrm{m}^{2}$ was created on Si tips by high-load scanning on a hard, synthetic diamond surface. The tip was then anodized in HF to create $100 \mathrm{~nm}$ diameter pores in this flat surface. Next, iron was electrochemically deposited into the pores to form catalyst particles. Tips prepared in this way were heated in low concentrations of ethylene at $800^{\circ} \mathrm{C}$, which is known to favor the growth of thin nanotubes (Hafner et al., 1998). When imaged by SEM, nanotubes prepared in this way were found to grow perpendicular to the surface from the pores as desired (Figure 5). Moreover, TEM observations revealed thin individual multiwalled nanotubes protruding from the ends of the silicon tips, with typical radii ranging from 3 to $5 \mathrm{~nm}$ (Figure 5). It is also
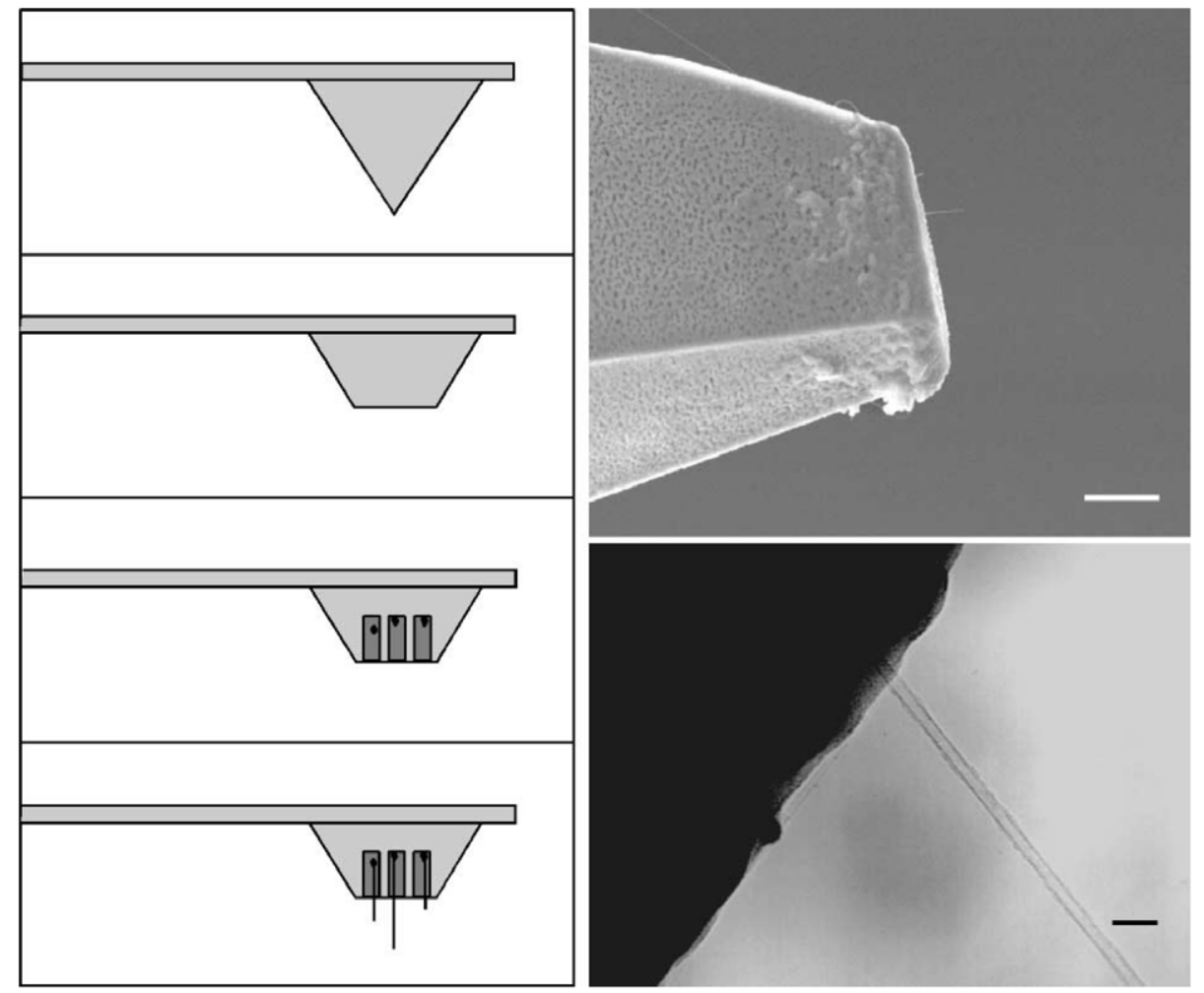

Figure 5. The pore-growth technique for CVD nanotube tip production: The panels on the left illustrate the steps involved in tip production: flattening, pore formation, catalyst deposition, and CVD nanotube growth. SEM (top right, $1 \mu \mathrm{m}$ scale bar) and TEM (bottom right, $20 \mathrm{~nm}$ scale bar) of individual carbon nanotube tips produced by the pore-growth method (Hafner et al., 1999a). 
worth noting, if tubes did not grow in an acceptable orientation or when they ultimately failed, that the carbon could be removed by oxidation and then CVD repeated to grow new nanotube tips (Hafner et al., 1999a).

These "pore-growth" CVD nanotube tips exhibit the favorable mechanical and adhesion properties found earlier with manually assembled nanotube tips, that is, the CVD tips buckled elastically, were very robust in imaging, and had low tip-sample adhesion. In addition, the ability to produce reproducibly thin, individual nanotube tips has enabled improved resolution (Hafner et al., 1999a) on isolated proteins. Continued development of the pore-growth nanotube tips has focused on the development of more controlled catalysts, such as well-defined iron oxide nanocrystals, to enable greater control over nanotube growth. In particular, this effort has enabled the controlled growth of thin SWNT bundles of 1-3 nm in diameter from pores made at the silicon tip ends (Cheung et al., 2000b). The pore-growth method has demonstrated the great potential of CVD to grow directly controlled diameter nanotube tips, although it still has some limitations. In particular, the preparation of a porous layer can be time consuming and may not place individual SWNTs at the optimal location on the flattened apex. One solution to this issue involves using focused ion beam milling, which can be carried out with 5-7 nm resolution, to create a single pore at the tip apex, which would then necessitate growth from only this one optimal position. This approach could also be automated on a wafer wide scale using commercial ion milling instruments.

\subsubsection{Surface growth}

An elegant yet simple alternative to produce CVD SWNT tips involves direct growth of SWNTs tips from the pyramid of a silicon cantilever-tip assembly. In this "surface growth" approach, we utilize the trade-off between the energy gain of the nanotube-surface interaction and energy cost to bend nanotubes to grow reproducibly SWNTs from the silicon pyramid apex in the ideal orientation for high-resolution imaging. This perhaps unintuitive result can be understood by considering the fate of a nanotube during growth. When a growing nanotube reaches an edge of the pyramid, it can either bend to align with the edge or protrude from the surface. The pathway followed by the nanotube is determined by a trade-off in the energetic terms introduced above: if the energy required to bend the tube and follow the edge is less than the attractive nanotube-surface energy, then the nanotube will follow the pyramid edge to the apex; that is, nanotubes are steered towards the tip apex by the pyramid edges. At the apex, the nanotube must protrude along the tip axis since the energetic cost of bending is too high. A schematic of this approach is shown in Figure 6.

This steering of nanotubes to the pyramid apex to form ideal probe tips has been demonstrated experimentally (Hafner et al., 1999b). For example, SEM investigations of nanotube tips produced by the surface growth method show that a very high yield of tips contains nanotubes only at the apex, with very few protruding elsewhere from the pyramid (Figure 6). These tips, which have been produced under conditions expected to yield SWNTs (Hafner et al., 1998), are sufficiently small that SEM cannot provide an accurate measure of their diameters. TEM analysis was able to demonstrate that the tips consist of individual SWNTs and small SWNT bundles. In the case of the small SWNT bundles, the TEM images show that the bundles are formed by nanotubes coming together from different edges of the pyramid to join at the apex, thus confirming the surface growth model described above (Figure 6). We 

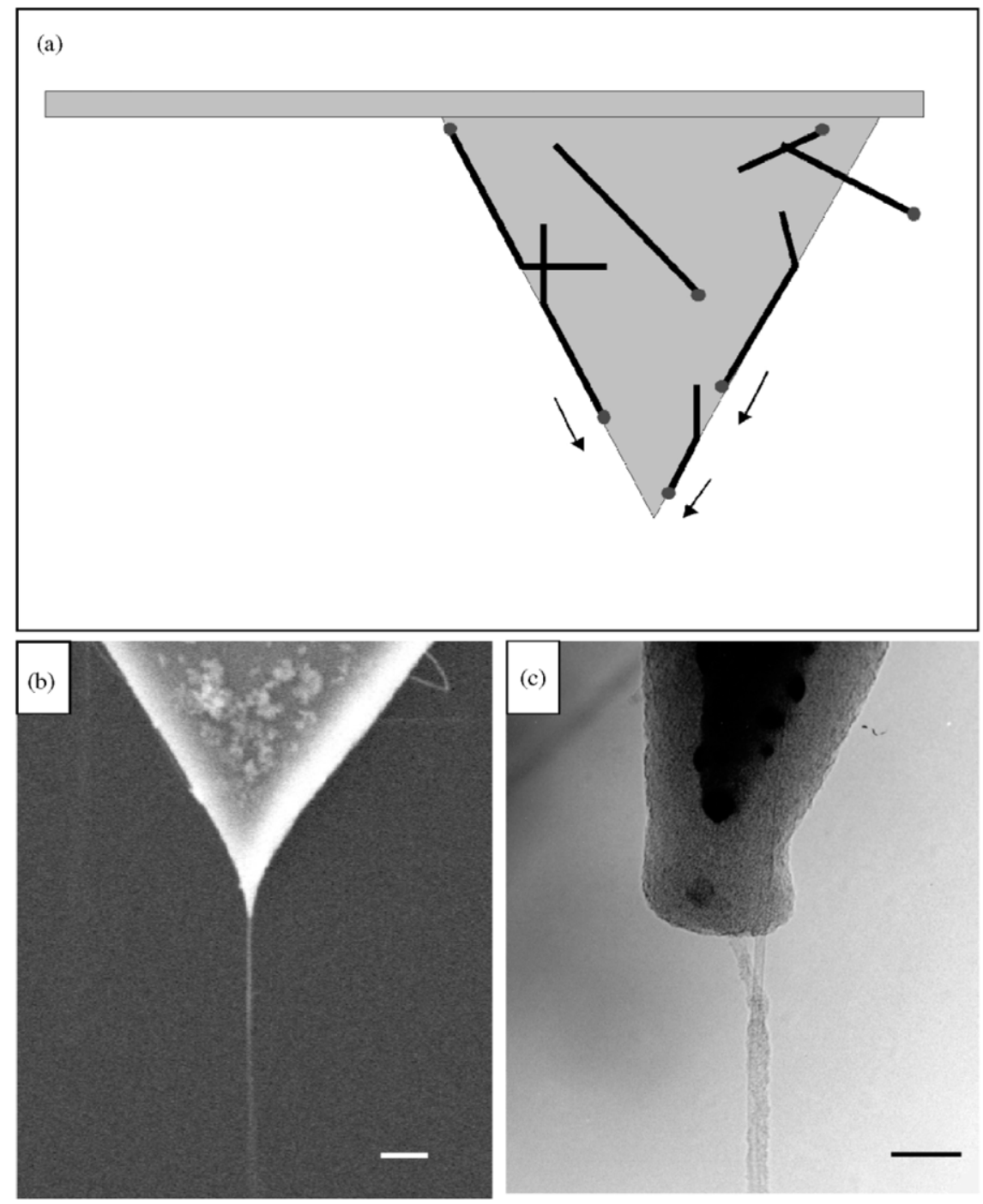

Figure 6. Surface growth method for CVD nanotube tip preparation: (a) Schematic illustration of surface growth process, where nanotubes grow on the pyramidal surface, guided along the edges towards the tip apex. (b) SEM and (c) TEM images of an SWNT surface growth tip consisting of two SWNTs (Hafner et al., 1999b). The scale bars in (b) and (c) are 200 and $20 \mathrm{~nm}$, respectively.

also believe that the surface-growth approach is especially important since it provides a conceptual and practical framework for preparing individual SWNT tips by lowering the catalyst density on the surface such that only 1 nanotube reaches the apex. Hence, by controlling reproducibly the catalyst density used for growth it is possible to produce well-defined individual SWNT tips, as shown in Figure 7 (Hafner and Hafner et al (2000a); Cheung et al., 2000c). Lastly, we note that these surface growth SWNT 


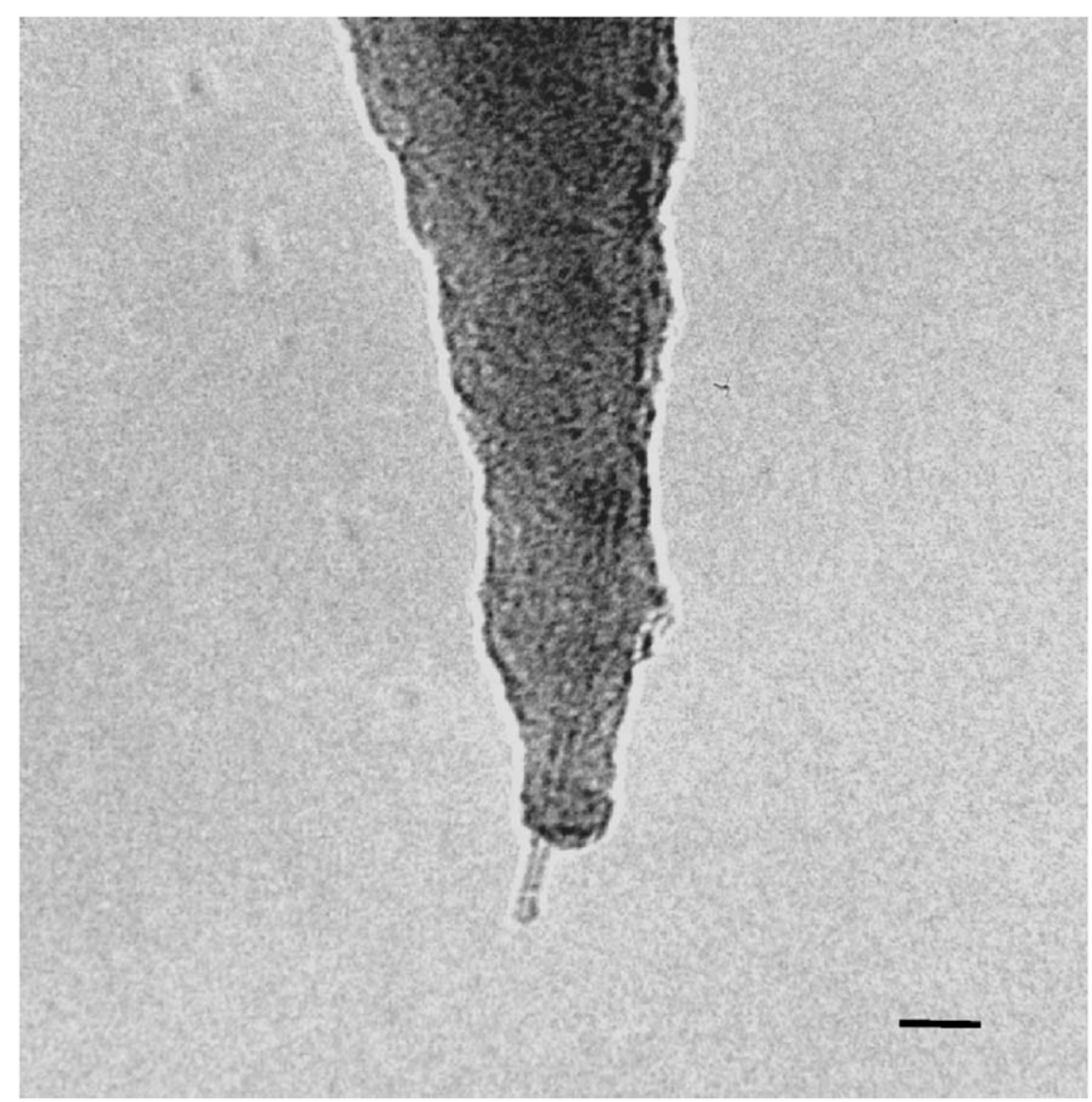

Figure 7. TEM image of an individual SWNT tip produced by controlling carefully the catalyst density (Cheung et al., 2000c). The scale bar equals $10 \mathrm{~nm}$.

tips display the elastic buckling behavior characteristic of other nanotube tips, and can be recycled by oxidation as with the pore-growth tips.

The synthesis of carbon nanotube AFM tips by CVD clearly resolves the major limitations of nanotube tips that arise from the manual assembly method. Rather than requiring tedious micromanipulation for each tip, it is possible to envision production of an entire wafer of SWNT tips. In addition to ease of production, CVD yields thin, individual SWNT tips that cannot be made by other techniques and perhaps represent the ultimate AFM probe for structural biology.

\section{Imaging with and resolution of nanotube probe tips}

\subsection{Evaluation of nanotube tip resolution with gold nanoparticles}

To accurately assess the structure and resolution of nanotube tips, an imaging standard is needed. Monodisperse gold nanoparticles, which are essentially incompressible in AFM, are ideal for this pur- 
Table 1. Comparison of tip radii of different types of carbon nanotube probes

\begin{tabular}{lc}
\hline Nanotube type & ${\text { Tip radius }(\mathrm{nm})^{\mathrm{a}}}^{\mathrm{a}}$ \\
\hline Mech. assembled MWNT & $\geq 6$ \\
Mech. assembled SWNT & $\geq 3.5$ \\
Pore-growth MWNT & $3.5-6$ \\
Pore-growth SWNT & $2-4$ \\
Surface-growth SWNT & $2-4$ \\
Low density surface growth $^{\mathrm{b}}$ & $\leq 2$ \\
\hline
\end{tabular}

${ }^{\text {a }}$ Tip radii were determined from images of $5.7 \mathrm{~nm}$ gold nanoparticles using the two-sphere model (Bustamante et al., 1993).

${ }^{\mathrm{b}}$ The lower limit of tips produced by this method is expected to be on the order of $0.35-0.5 \mathrm{~nm}$.

pose (Vesenka et al., 1993). We have used $5.7 \mathrm{~nm}$ diameter gold nanoparticles to characterize different types of carbon nanotube tips. This size of gold nanoparticle represents an appropriate one for testing tips that will be used for imaging large isolated proteins, which have been the focus of our work. The effective tip radius is calculated from the particle image using the two-sphere model (Bustamante et al., 1993). Manually assembled MWNT tips typically have shown radii as small as $6 \mathrm{~nm}$, which is expected for the size of arc-produced MWNT material (Wong et al., 1998a). Manually assembled SWNT tips are composed of thick SWNT bundles. Since the SWNT bundles are made from $1.4 \mathrm{~nm}$ nanotubes, the etching procedure would at times produce high resolution on gold nanoparticles, down to 3 nm radius (Wong and Wong). Pore-growth CVD MWNT have radii ranging from 3 to $6 \mathrm{~nm}$ when measured from gold nanoparticle images (Hafner et al., 1999a). These tips provide better resolution than manually assembled MWNT tips and typical assembled SWNT tips, because the CVD MWNT tips are relatively thin and always consist of individual nanotubes. Both the pore-growth (Cheung et al., 2000b) and surface growth (Hafner and Hafner et al (2000a); Cheung et al., 2000c) SWNT tips have shown radii of less than $4 \mathrm{~nm}$, reflecting the very thin SWNT bundle or individual SWNT tip structures possible with these methods. These results are summarized in Table 1.

Another very significant point with the nanotube tips is that the range of tip radii measured by AFM agreed with the range measured by TEM. This demonstrates that, unlike in the case of microfabricated $\mathrm{Si}$ and $\mathrm{Si}_{3} \mathrm{~N}_{4}$ tips, we are imaging with very well-defined probes, and that the probe contribution to images can be readily removed by deconvolution techniques. Lastly, we reiterate that the levels of resolution achieved with the nanotubes were obtained on gold nanoparticles nearly $6 \mathrm{~nm}$ in height, rather than on 1-2 nm features which at times indicate high resolution with tenuous, asperities on microfabricated $\mathrm{Si}$ and $\mathrm{Si}_{3} \mathrm{~N}_{4}$ tips.

\subsection{Studies of well-characterized biological structures}

To determine the potential of nanotube tips in structural biology, DNA and several well-characterized proteins have been imaged. DNA was imaged by manually assembled MWNT tips in air (Nishijima et al., 1999) and in fluid (Li et al., 1999). The fluid imaging experiments produced a measured 
height of $2 \mathrm{~nm}$, the expected value based on the intrinsic DNA diameter, and the resolution in these studies was on the order of 3.5-5 nm. These values for the resolution are consistent with that expected for multiwalled nanotube material, but also similar to the best values observed with microfabricated tips (Han et al., 1997; Lyubchenko and Schlyakhtenko, 1997). However, as discussed above, we do not believe that imaging DNA provides a robust test of resolution and capabilities of tips that will be used for investigating relatively large proteins.

Studies of isolated proteins provide a much more stringent test of the capabilities of probe tips, and we believe demonstrate clearly the advantages of nanotube tips. Pore-growth MWNT CVD tips were used to image isolated IgG and Immunoglobulin-M (IgM) antibody proteins (Hafner et al., 1999a; Cheung et al., 2000b). IgG is a ca. $180 \mathrm{kDa}$ molecule with a characteristic Y-shaped structure approximately $15 \mathrm{~nm}$ across as determined by single crystal X-ray diffraction measurement (Silverton et al., 1977). Previous AFM imaging studies either saw no structure (Bergkvist et al., 1998), a heart-shaped structure (Fritz et al., 1997), or the Y-shape only at cryogenic temperatures (Zhang et al., 1996). In our studies, the Y-shaped structure was easily and reproducibly resolved by CVD MWNT tips at room temperature as shown in Figure 8 (Cheung et al., 2000b). These IgG images are also compared (Figure 8) with the crystal structure to emphasize the very small amount of tip-induced broadening in the observed images obtained with nanotube tips.

Pore-growth CVD MWNT tips have also used to examine the structure of IgM (Hafner et al., 1999a). This antibody is a ca. 1 MDa protein consisting essentially of 5 IgGs arranged in a pentameric structure. To date, it has not been possible to crystallize IgM and determine its atomic structure by X-ray diffraction methods. Previous structural analysis has relied primarily on electron microscopy analysis, which has elucidated the basic features of the pentameric structure (Perkins et al., 1991). Our room temperature studies with the pore-growth CVD MWNT nanotube tips showed clearly the pentameric structure, including five external pairs of antigen binding fragments (Fab domains) and five internal Fc fragments (Figure 9). In addition, these images occasionally exhibited the loop structure connecting two of the five Fc domains that likely corresponds to the joining (J) loop (Hafner et al., 1999a). Because the exact structure of this region is still unclear (Perkins et al., 1991), our nanotube AFM investigations have shown clearly the potential of nanotube probes to reveal new structural features on large proteins that cannot be crystallized for diffraction studies.

To test the resolution of the smaller diameter CVD SWNT tips, we have focused a smaller protein GroES. GroES, which is a component of the GroEL/GroES chaperonin system involved in protein folding, is a hollow dome-shaped heptamer that is approximately $8 \mathrm{~nm}$ in outer diameter (Sigler et al., 1998). The seven $10 \mathrm{kDa}$ subunits each consist of a core $\beta$-barrel with a $\beta$-hairpin loop at the top and bottom. The top $\beta$-hairpins point inward to form the top of the dome, while the bottom hairpins are disordered when not in contact with GroEL (Hunt et al., 1996). Previously, AFM images of GroES have only been obtained on densely packed arrays in contact mode (Mou et al., 1996). In this work, subunit resolution was observed when the samples were fixed using gluteraldehyde but lower resolution was obtained in the absence of fixation. In our studies (Cheung et al., 2000b), CVD SWNT tapping mode images of unfixed individual, well-separated GroES molecules on mica reveal two conformations (Figure 10). One conformation shows a ring-like structure with an $11 \mathrm{~nm}$ outer diameter and internal structure to the ring. The other conformation simply looks like a dome of the same diameter. We have interpreted these two conformations as the two sides of the GroES heptamers. The domed image is the 


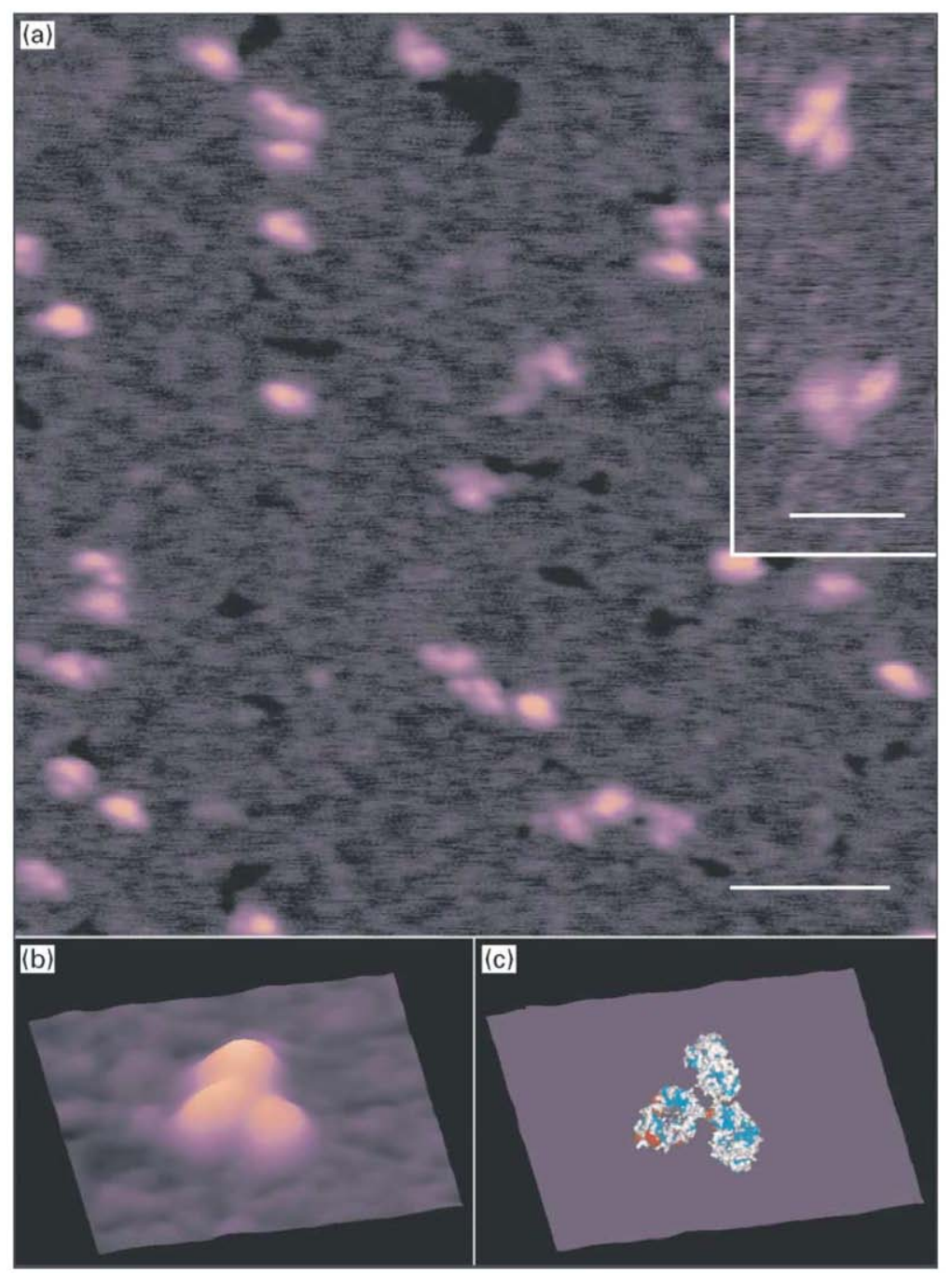

Figure 8. Nanotube tip AFM images of Immunoglobulin G: (a) A field of several IgG molecules highlights the flexibility of the molecule, which leads to many conformations on the surface (scale bar equals $50 \mathrm{~nm}$ ). The inset (scale bar equals $20 \mathrm{~nm}$ ) shows two molecules exhibiting the well-defined Y-shaped structure expected for this protein. (b) Three-dimensional plot of an isolated IgG molecule, which exhibits the characteristic Y-shaped structure, compared to (c) the crystal structure to emphasize the minimal tip-induced broadening (Cheung et al., 2000b).

side with the ordered $\beta$-hairpins creating the dome roof, and the ringed image is the disordered $\beta$-hairpins open to the hollow cavity. The disordered hairpins have conformational flexibility, but it is possible to observe the expected seven-fold symmetry (Hunt et al., 1996) as shown in Figure 10b. For comparison, Figure 10c shows the bottom view of the GroES crystal structure with the top of the dome removed to make the pore visible. These results demonstrate the ability of the present CVD nanotube tips to achieve sub-molecular resolution on isolated protein assemblies without fixing, and thus clearly suggest the possibility of high-resolution in vitro work in the future. 


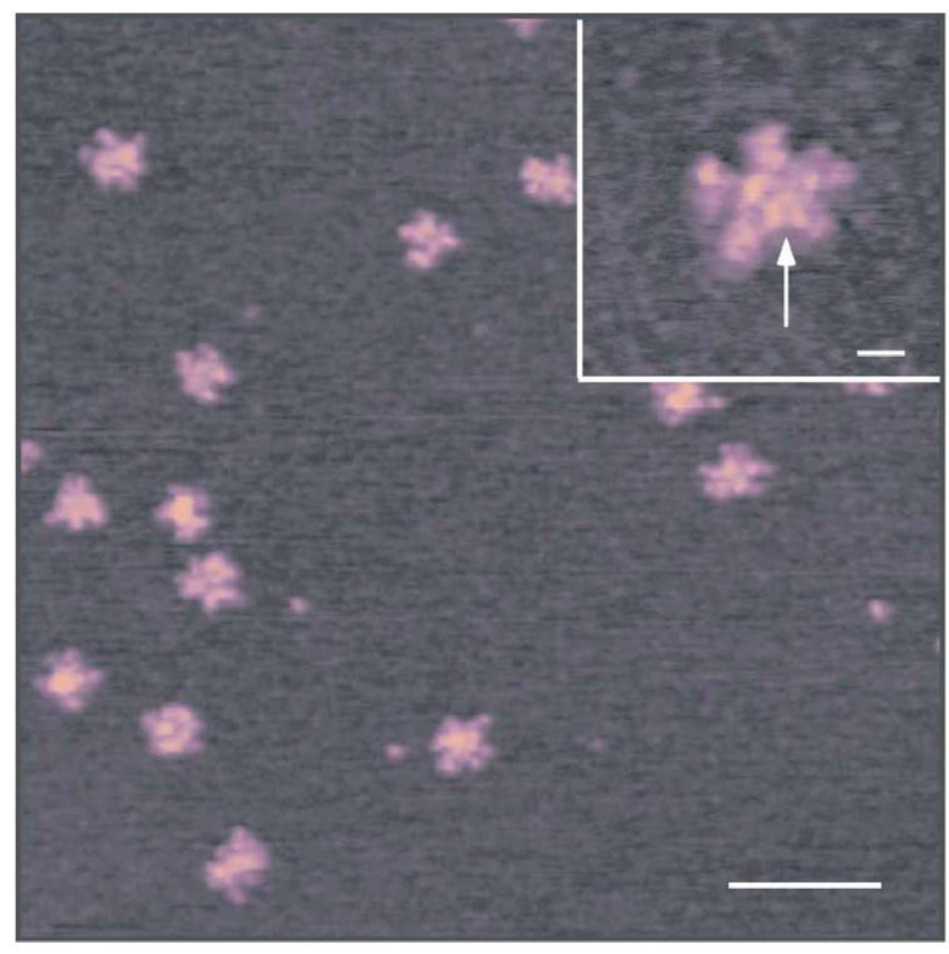

Figure 9. Nanotube tip AFM images of IgM: The pentameric structure of IgM is not always apparent due to the high conformational flexibility of this molecule (scale bar is $100 \mathrm{~nm}$ ). The inset shows an example with clear pentameric symmetry, including structural information on the central region (Hafner et al., 1999a). The inset scale bar equals $10 \mathrm{~nm}$. The arrow highlights the position of the J-loop.

\section{Structural biology and other applications of nanotube tips}

We believe that the CVD nanotube tips described above represent powerful probes for structural biology studies of systems that have not been characterized by other methods, since these tips provide reproducible and predictable resolution that is comparable to that obtained with cryoEM. While the very recent advances in the growth of the SWNT probes have not yet enabled extensive studies of complex protein and protein/nucleic acid systems, the early results described below, which address (1) intermediates along the aggregation pathway of amyloid proteins, (2) ATP-dependent nucleosome remodeling, and (3) DNA sequence determination, indicate clearly the potential of these structural probes.

\subsection{Aggregation and fibril formation of amyloid proteins}

Cortical amyloid plaques, comprising a fibrillar form of the amyloid- $\beta$ proteins $\mathrm{A} \beta 40$ and $\mathrm{A} \beta 42$, are a defining pathological feature of Alzheimer's disease (AD) brain (Lansbury, 1996; Selkoe, 1999), and moreover, the number of plaques at autopsy roughly correlates with the severity of AD symptoms (Cummings et al., 1996). These and other pathological observations suggest that amyloid fibril forma- 


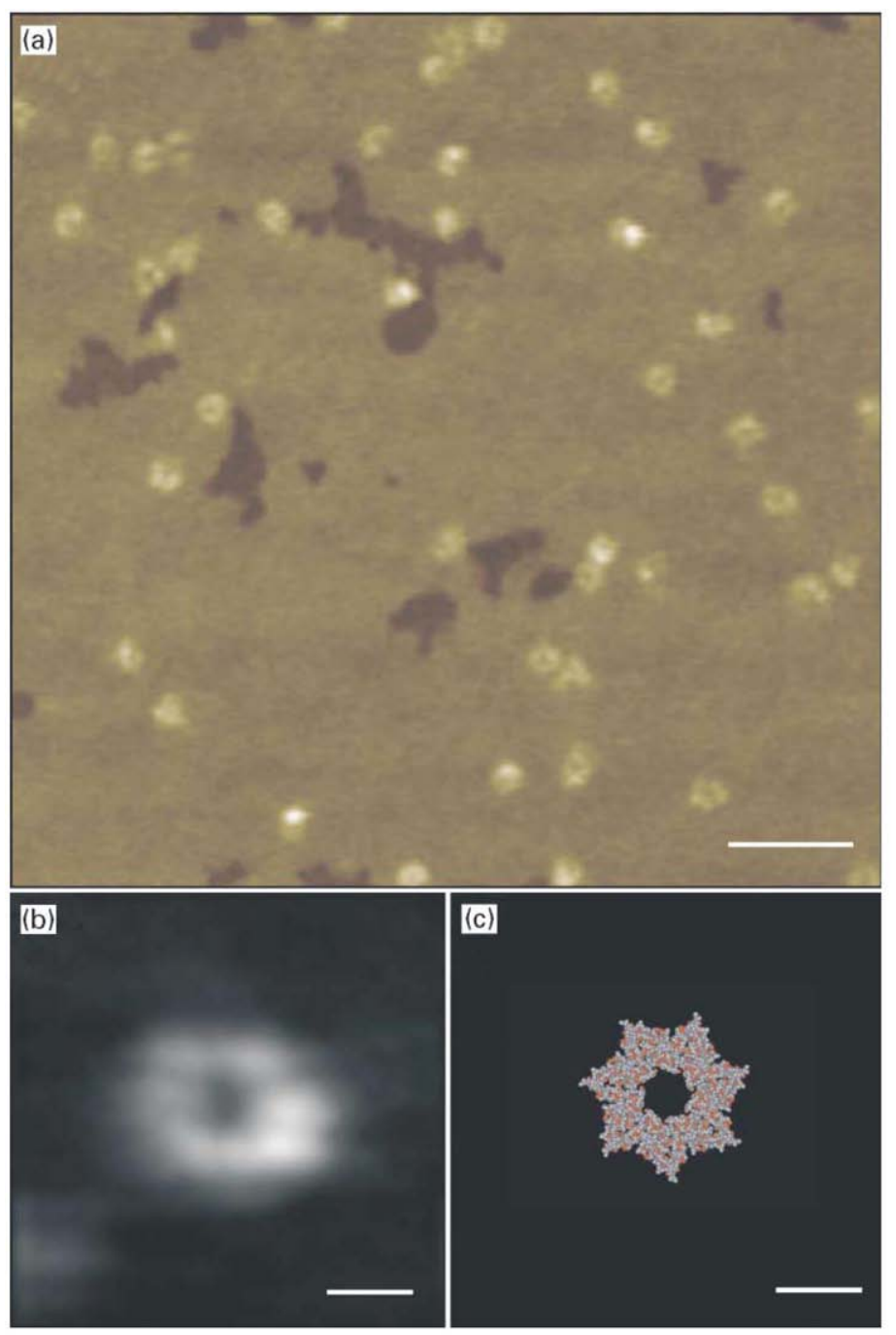

Figure 10. Nanotube tip AFM images of isolated GroES molecules: (a) Field of many GroES molecules reveal dome and ring conformations, representing the two sides of GroES heptamer (scale bar equals $50 \mathrm{~nm}$ ). (b) A high-resolution image of the ring conformation shows clearly the heptameric symmetry, and little tip-induced broadening relative to the crystal structure (c) (Cheung et al., 2000b). The scale bars in (b) and (c) equal $5 \mathrm{~nm}$.

tion is an important event in the AD process (Cummings et al., 1996; Lemere et al., 1996), although it is not known whether the fibrils or a precursor intermediate is responsible for neuronal cell death. Understanding this latter point is especially important since therapeutic agents designed to inhibit fibril formation at too late a stage could result in the build up of an intermediate that accelerates the onset of AD. In this regard, it is clearly important to define the pathway of fibrillization for monomeric $\mathrm{A} \beta$ proteins, including the structural characterization of intermediates. 

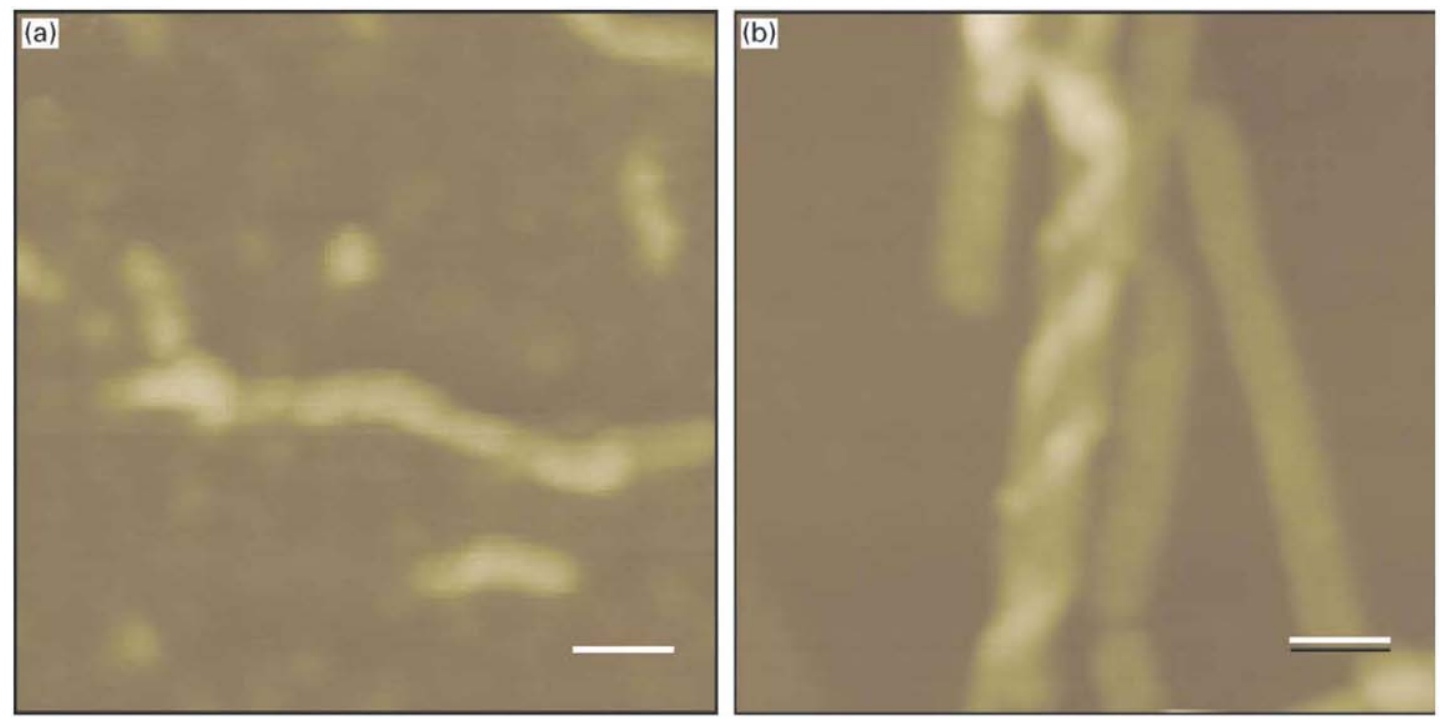

Figure 11. High-resolution nanotube tip images of $\mathrm{A} \beta$ aggregation: Images of (a) protofibrils and (b) helical fibrils and rigid filaments. The scale bars in (a) and (b) are $25 \mathrm{~nm}$.

AFM represents a powerful tool for studying the fibrillization pathway since the wide range and coexistence of relatively large structural species are difficult to probe using diffraction and NMR methods (Harper et al., 1997). Our previous studies of $\mathrm{A} \beta$ fibril formation revealed the existence of a discrete, dynamic intermediate, which we termed the amyloid protofibril (Harper and Harper). Oligomeric species which are probably the same as the protofibrils, have also been observed by EM (Walsh et al., 1997). Images of A $\beta$ protofibrils and fibrils obtained with nanotube tips are shown in Figure 11 (Hafner et al., 2000b). These data show the same overall features as reported in our earlier studies using microfabricated silicon tips, although the structural resolution is higher. For example, the helical structure observed in fibrils is much clearer and more detailed in images recorded with SWNT tips.

In addition, $\mathrm{A} \beta$ oligomers, which are smaller than typical protofibrils, were observed in previous AFM studies (Harper and Harper; Lambert et al., 1998). Height measurements made on these oligomers were interpreted as diameters of a globular morphology, due to the resolution limitations of conventional tips. To obtain greater structural information about these and other potentially pathogenic $\mathrm{A} \beta$ species that precede and/or coexist with protofibrils, we have studied the early stages of fibrillization using high-resolution CVD SWNT tips. Images of the A $\beta 40$ protein obtained with SWNT probes after 1-3 days of incubation show a new structural species with a 5-6 nm height, a ca. $19 \mathrm{~nm}$ diameter and fourfold internal structure as shown in Figure 12 (Hafner et al., 2000b). The height of this species is similar to that found in previous low-resolution studies, where it was assumed to correspond to a globular structure. Our higher resolution images with nanotube tips exhibit clear four-fold symmetry and a central depression ca. $1 \mathrm{~nm}$ deep. This depression could correspond to a pore through the center of the molecule that is too small to be accessed by the tip. Based on these structural features we have termed this new morphology the annular $\mathrm{A} \beta$ structure. 


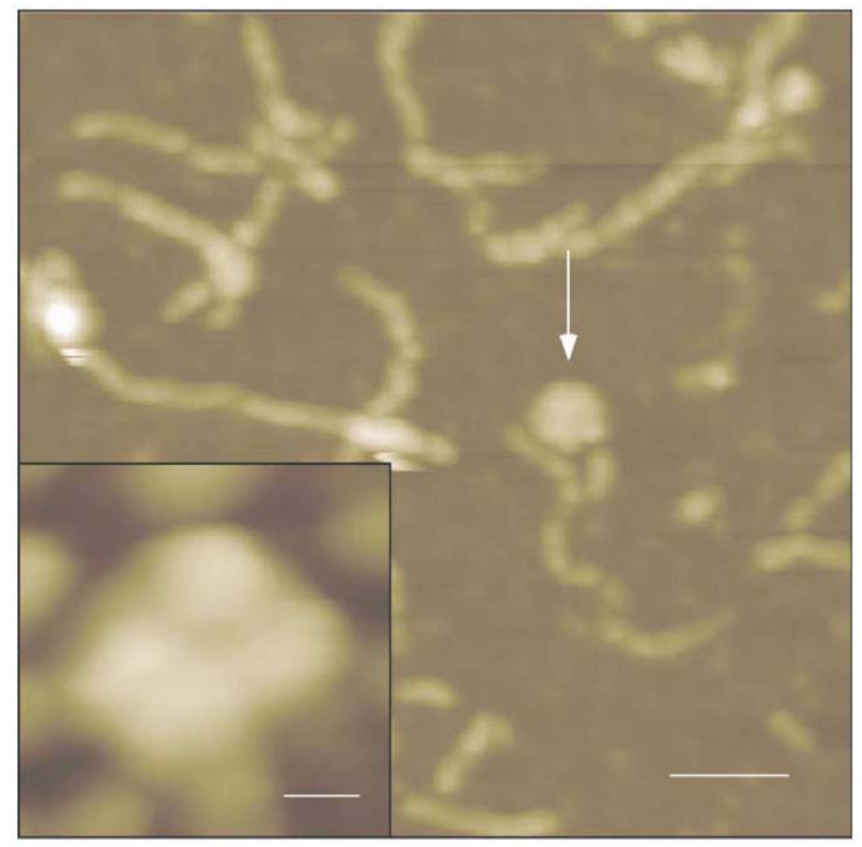

Figure 12. Nanotube tip AFM images of the $\mathrm{A} \beta$ annular structure: The annular structure, highlighted by the white arrow, is seen among protofibrils deposited on mica (scale bar is $50 \mathrm{~nm}$ ). The inset shows a high-resolution image of an $\mathrm{A} \beta$ annular structure (scale bar equals $5 \mathrm{~nm}$ ).

We have outlined the possible relationship of the annular $\mathrm{A} \beta$ structure to other species characterized along the fibrillization pathway in Figure 13 (Hafner et al., 2000b). The four-fold morphology of the annular structure is qualitatively similar in appearance to the subunits of elongated protofibrils, and thus it is reasonable to speculate that the annular structure is a head-to-tail loop of a short protofibril. However, this head-to-tail mechanism is hard to reconcile with the fact that (1) the annular $\mathrm{A} \beta$ structure has a constant $18-21 \mathrm{~nm}$ diameter, while protofibrils greater than $200 \mathrm{~nm}$ in length are commonly observed, and (2) the heights of the annular structure, $5.8 \mathrm{~nm}$, and protofibril, $4-5 \mathrm{~nm}$, are recognizably different. Hence, we have suggested that the molecular mechanisms for formation of the annular structure and protofibrils are distinct.

The annular $\mathrm{A} \beta$ structure may be relevant to $\mathrm{AD}$ pathology for several reasons. The possibility that nonfibrillar $\mathrm{A} \beta$ oligomers may be pathogenic has been suggested by several experiments. Nonfibril$\operatorname{lar} \mathrm{A} \beta$-derived species have been shown to have in vitro neurotoxic activity (Lambert et al., 1998) and may be responsible for neurodegeneration in AD (Hsia et al., 1999; Moechars et al., 1999). In addition, there is evidence that $\mathrm{A} \beta$ forms ion channels in lipid bilayers (Arispe et al., 1993; Lin et al., 1999) and this channel activity has been implicated in AD pathogenesis (Arispe et al., 1993). It is interesting to suggest that the annular $\mathrm{A} \beta$ structure is this proposed channel forming structure. As precedent, a recent cryoEM study of the membrane-active $\alpha$-latrotoxin neurotoxin reveals a similar structure: a $15-18 \mathrm{~nm}$ diameter tetramer that contains a $1 \mathrm{~nm}$ diameter ion channel (Orlova et al., 2000). Our current efforts 


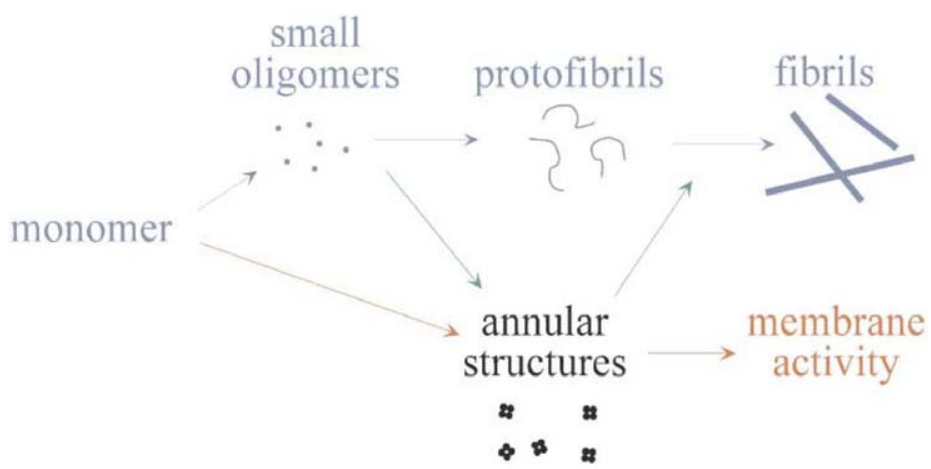

Figure 13. A $\beta$ aggregation pathways: The blue pathway represents that observed with standard AFM tips in which featureless oligomers lead to protofibrils, which ultimately form fibrils. The $\mathrm{A} \beta$ annular structure, which is shown on the red pathway, may exhibit distinct pathological activity or could return to the fibril pathway (green arrow).

are focused on isolating the annular $\mathrm{A} \beta$ structure so that its structure can be further characterized and its potential biological activity studied.

\subsection{Nucleosome remodeling}

Eukaryotic cells package their DNA by complexing it with a defined set of histone proteins. Approximately 146 base pairs of DNA wrap around an octamer of histones, composed of an H3-H4 histone tetramer and two H2A-H2B histone dimers, thereby forming a DNA-protein nucleosome structure (Figure 14a). Additional structuring of the nucleosome arrays by linker histones produces the higher order chromatin structure that serves to further compact the DNA (Kornberg and Lorch, 1999; Zlatanova et al., 1998). Fundamental cellular processes such as DNA replication and gene transcription require the binding of transcription factor proteins to specific DNA sequences. In the past, the emphasis has been on understanding the interactions between specific protein transcription factors and naked DNA. However, it has become evident that histone and non-histone chromatin proteins can block access to DNA and thereby hinder replication. Hence, the cell must possess mechanisms to modify the accessibility of nucleosomal DNA to DNA binding factors. Two such critical mechanisms have been elucidated thus far. First, protein complexes that remodel chromatin structure and make the DNA more accessible can regulate activity (Kadonaga, 1998). Second, alterations in the chemical state of histones, which affect DNA-histone binding, can regulate activity (Hassig and Schreiber, 1997). As an example of the first mechanism, the 2 MDa protein complex SWI/SNF has been shown to possess ATP-dependent nucleosome remodeling activity (Pazin and Kadonaga, 1997; Schnitzler et al., 1998), while the recently discovered NuRD complex exhibits both ATP-dependent remodeling and deacetylation activity (Hassig and Schreiber, 1997).

Thus far, several homologous remodeling factors have been characterized. These are nucleotide triphosphate binding proteins that have conserved helicase/ATPase domains. Hydrolysis of ATP appears to be necessary to produce the energy needed to disrupt nucleosome structure. These remodeling factors have been shown to function in a variety of cellular processes, including the regulation of gene expression. For example, in yeast, the SWI/SNF chromatin remodeling complex is required to achieve tran- 

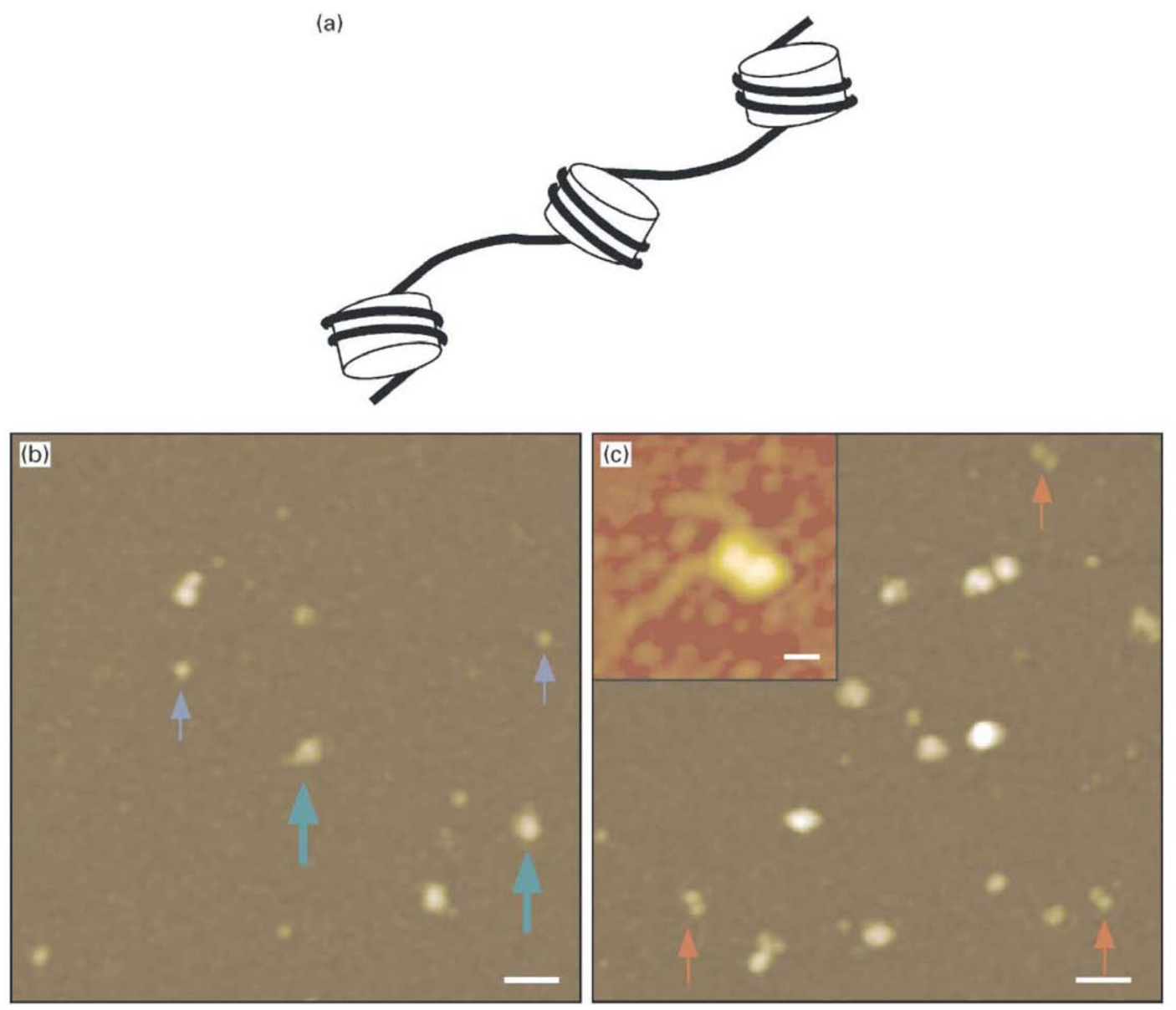

Figure 14. Nucleosome remodeling: (a) Schematic representation of chromatin showing histone octamers (open cylinders) and DNA (solid black line). The nucleosome corresponds to the histone octamer wrapped with DNA ca. 146 base pairs long. CVD MWNT tip images of (b) mononucleosome and hSWI/SNF without ATP, and (c) mononucleosome and hSWI/SNF in the presence of ATP. The blue and green arrows in (b) highlight mononucleosome and SWI/SNF complex; the red arrows in (c) highlight the dimer product. The scale bars in (b) and (c) are $50 \mathrm{~nm}$. The inset in (c) is a high-resolution image of the dimer product; scale BAR $=10 \mathrm{~nm}$.

scription of a group of inducible genes. The RSC complex is essential for yeast growth. In Drosophila, the activity of the NURF complex is required for the binding of certain transcription factors to DNA. Each of these complexes contains SWI/SNF homologues which can disrupt nucleosomal structure in an ATP-dependent manner, as determined by increased accessibility to DNA cleaving enzymes and improved binding of transcription factors. However, the exact mechanism by which nucleosome structure is altered remains unclear (Pazin and Kadonaga, 1997).

Two recent reports have provided biochemical insight into the mechanism of chromatin remodeling (Lorch et al., 1998; Schnitzler et al., 1998). It has been demonstrated that the SWI/SNF and RSC chromatin remodeling complexes can produce a stable altered nucleosome structure. This altered form is ap- 
proximately twice the size of a single mononucleosome, contains only the core histones and DNA, and has an increased susceptibility to cleavage by DNase I and restriction enzymes. It has been suggested based on biochemical evidence that the remodeled state may represent a "dimerized" form of mononucleosomes, although clearly there exists a need for direct structural information. In addition, there is very little understanding of the relationship of the remodeled model system "dimer" to remodeling in polynucleosomes and chromatin. For example, do adjacent or distant nucleosome units become dimerized or are there new structural changes in polynucleosomes. High-resolution SWNT AFM imaging represents a promising approach for obtaining much needed structural information on these large systems.

Our initial efforts have focused on using AFM to examine structurally the proposal that the human SWI/SNF (hSWI/SNF) remodeling of mononucleosomes creates a dimer with altered DNA-histone contacts (Schnitzler et al., 1998). To address carefully this problem we have used CVD nanotube probes to characterize mononucleosomes and dinucleosomes isolated from human HeLa cells and the products obtained from the reaction of hSWI/SNF and mononucleosomes with and without ATP (Hafner et al., 2000c). Images characteristic of the products obtained from the reaction in the absence and presence of ATP are shown in Figure 14. These data suggest that no structural changes occur in the absence of ATP, while dimer products are observed in the presence of ATP. While these observations are expected on the basis of biochemical studies, we believe they also provide a taste of what can be expected in the future as one exploits higher resolution probes and investigates remodeling of polynucleosomes.

In addition, these studies of mononucleosome remodeling have shown substructure in the hSWI/ SNF complex. An image, which was obtained with a CVD MWNT tip, is shown in Figure 15. The images show that the SWI/SNF complex is a multilobed (subunit) structure, and appears to have a 2-fold axis (Hafner et al., 2000c). Our ability to resolve substructure in SWI/SNF, which was achieved even without the current, higher resolution CVD SWNT tips, opens up a number of possibilities for future studies. These opportunities include (1) probing for distinct conformational states in this ATP-dependent machine and (2) using functional imaging to probe for DNA and/or histone binding sites.

\subsection{DNA sequence analysis and haplotyping}

The reproducibly high resolution afforded by SWNT probe tips can also open up unexpected opportunities, and in this regard we have investigated their possible uses in DNA sequence analysis and related genomics problems (Woolley et al., 2000). This effort is motivated by the recognition that the haplotype of a subject - the specific alleles associated with each chromosome homologue - is critical to maximizing the impact of single nucleotide polymorphism (SNP) mapping (Brookes, 1999). However, the current methods for determining haplotypes have significant limitations that have prevented their use in large-scale genetic screening. For example, parental genotyping can be used to infer haplotypes in a family study (Hodge et al., 1999; Sobel and Lange, 1996), although in many cases it is impractical or impossible to obtain parental DNA.

Our method utilizes high-resolution SWNT probes to read directly multiple polymorphic sites in DNA fragments containing from ca. 100 to at least 10,000 bases. The approach involves specific hybridization of labeled oligonucleotide probes to target sequences in DNA fragments, using polymerase to create double-stranded DNA around the probe, followed by direct reading of the presence and spatial 


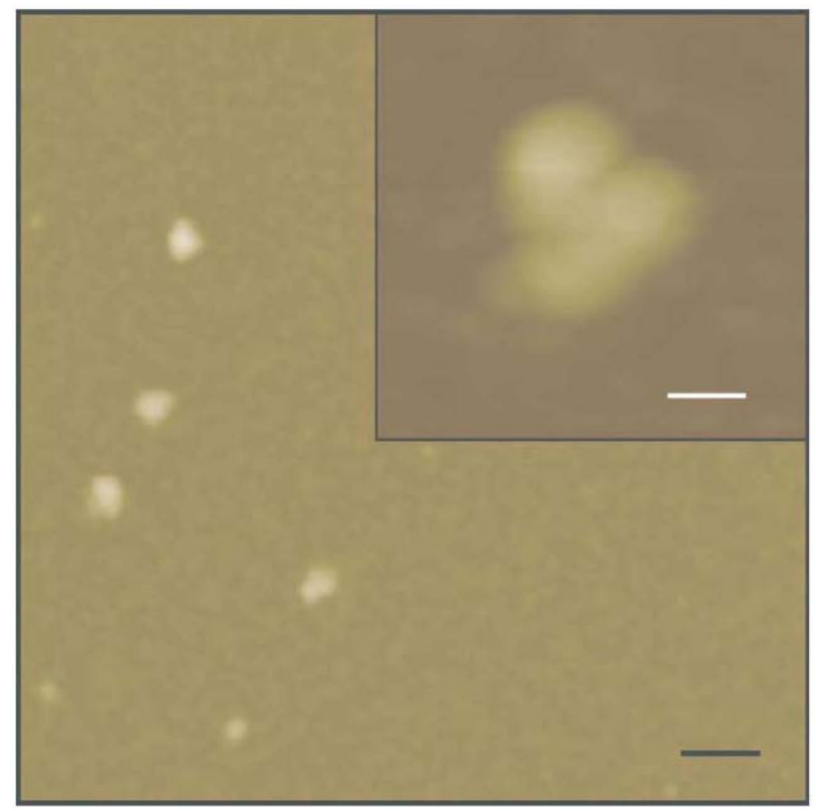

Figure 15. Nanotube tip AFM images of the hSWI/SNF complex reveal a multi-lobed structure (scale bar is $50 \mathrm{~nm}$ ). The inset shows a highresolution image of a single $\mathrm{hSWI} / \mathrm{SNF}$ complex (scale bar is $10 \mathrm{~nm}$ ).

locations of the labels by AFM (Figure 16). The oligonucleotide probes are designed such that, under appropriate hybridization conditions, binding does not occur in the presence of a single-base mismatch at polymorphic sites, i.e., labels are detected only at sequences fully complementary to the oligonucleotides. High-resolution SWNT probes are critical to our approach since they enable different size probes to be reproducibly distinguished as required for simultaneous detection of different polymorphic sites on single DNA fragments (Woolley et al., 2000). A similar approach may be possible using single molecule optical methods. However, an optical approach will require that fluorescent blinking and photobleaching of single labels be accounted for, while at the same time acquiring sufficient data to identify the centroid of a fluorescent peak to high accuracy.

Our new nanotube-AFM method has been tested by identifying the spatial location of specific sequences with excellent discrimination from corresponding single-base mismatches in the M13mp18 plasmid using seven base oligonucleotide probes. The essence of the experiments is shown in the image of a DNA molecule that was marked with a streptavidin-labeled GGGCGCG sequence (Figure 17a). This image shows a DNA fragment with a $2200 \mathrm{~nm}$ contour length consistent with the 7249 base pairs of M13mp18, and a distinct streptavidin label $1080 \mathrm{~nm}$ from one end of the Bgl II digested DNA. Histogram summaries of results obtained from a number of streptavidin-labeled M13mp18 DNA molecules showed clear peaks at 0.48 (3512 bp) and 0.40 (2893 bp) from the fragment ends, for samples cut with $\mathrm{Bgl}$ II and Bam HI, respectively. In contrast, control experiments with unlabeled oligonucleotides did not exhibit clusters of labels, indicating that the histogram peaks are due to specific detection of streptavidin. These results show that the GGGCGCG site is at base 3390 (Figure 17b), in good agreement with 


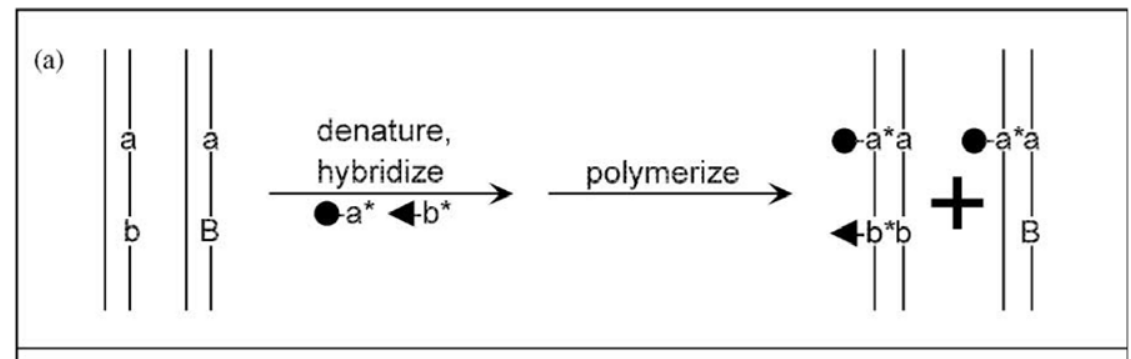

(b)

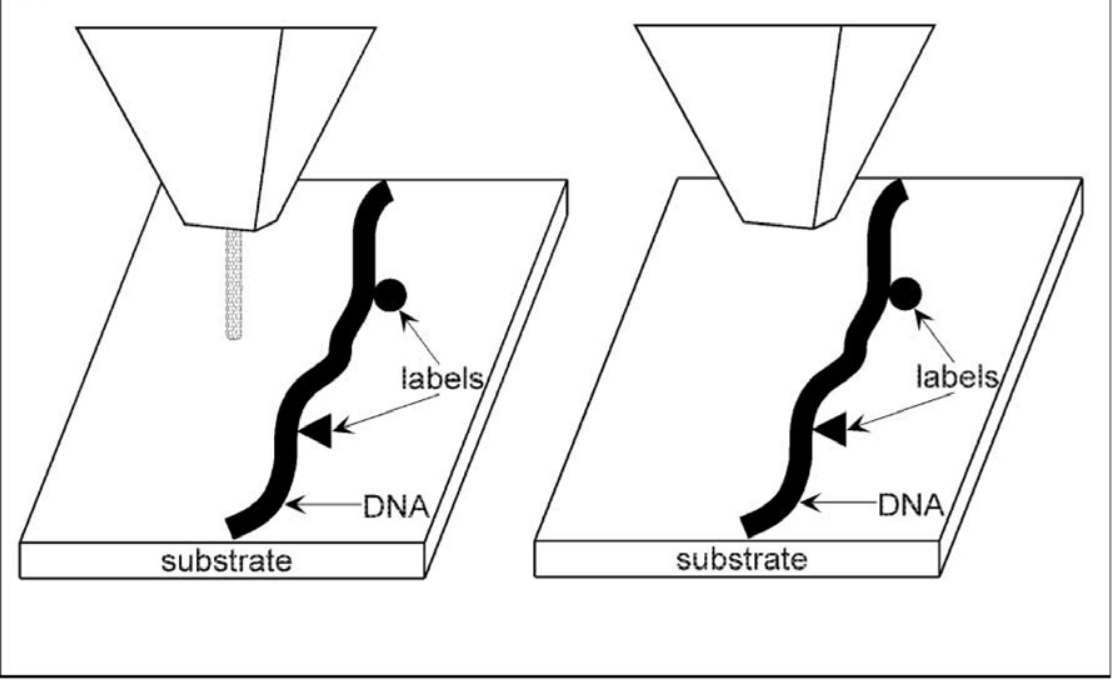

Figure 16. (a) Schematic illustration of the method for labeling specific DNA sites for detection with SWNT AFM probes. Labeled oligonucleotide probes $\left(\bullet-a^{*}\right.$ and $\left.<-b^{*}\right)$ are specifically annealed to their complementary target sequences $(\mathrm{a}$ and $\mathrm{b}$ ) but not to sequences with a single-base mismatch (A and B) in the single-stranded DNA template. DNA polymerase and dNTPs are then used to synthesize double-stranded DNA fragments specifically labeled at a and b with $\bullet$ and $\mathbf{4}$, respectively. (b) Schematic illustrating the reading of the labeled DNA with an SWNT tip (left) and an Si tip (right). Only the nanotube has sufficient resolution to distinguish reproducibly the different labels.

the known location (base 3405). In addition, these data exhibited no evidence for labeling at the singlebase mismatch sites located at 1115 and 3595, thus demonstrating the potential for SNP detection.

Because the streptavidin and IRD800 molecules can be readily distinguished on the basis of their heights and shapes using the SWNT tips, it is possible to detect two or more distinct sites using this method. Multiplexed detection has been tested by imaging M13mp18 labeled at GGGCGCG with IRD800 and at TCTCAGC with streptavidin using SWNT probes (Woolley et al., 2000). From histograms we have calculated that TCTCAGC occurs at bases 2024 and 4059, in good agreement with its known positions at 2013 and 4077, and that GGGCGCG is at base 3422, corresponding well with the expected value of 3405 . These results demonstrate clearly the potential for multiplexed sequence detection in large DNA strands and open the possibility for profiling multiple polymorphic sites on DNA fragments in the 10 kilobase or larger size range.

The model experiments carried out on M13mp18 suggested that this approach could be used for identifying specific haplotypes associated with genetic disorders. We have demonstrated this critical 
(a)

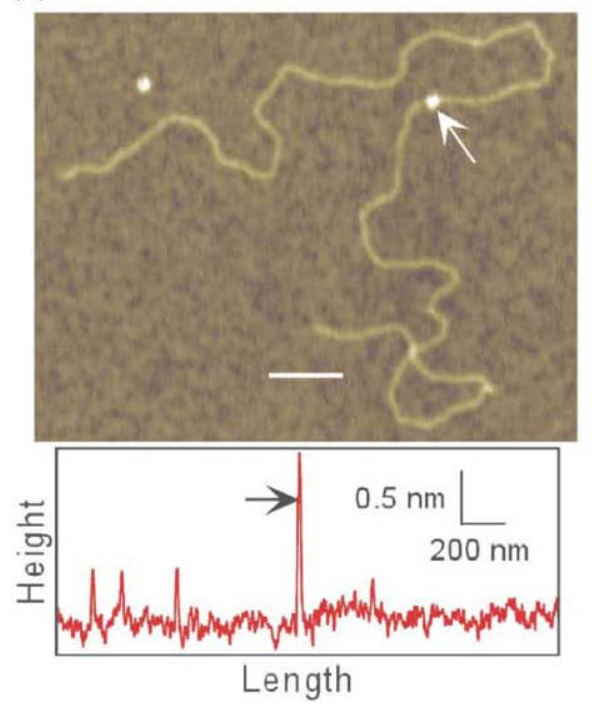

(b)

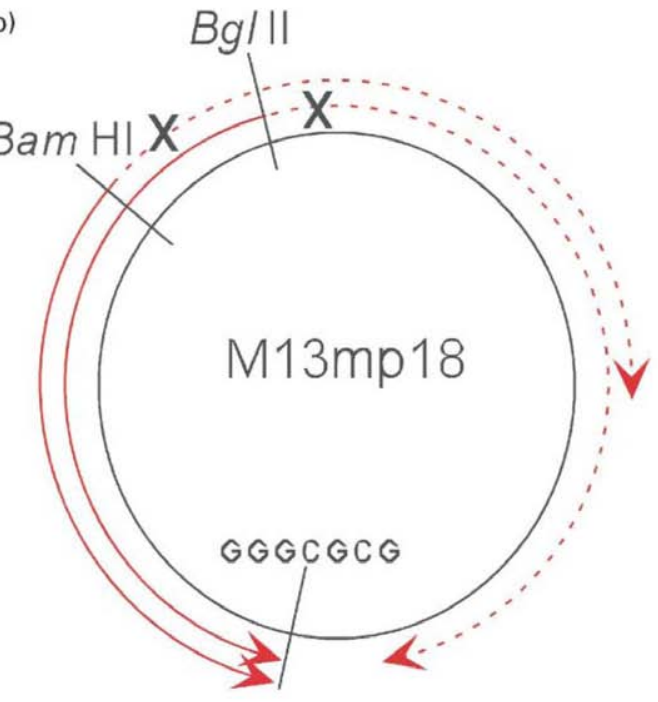

Figure 17. Detection of labeled DNA sites with nanotube tips: (a) SWNT tip image and height profile along DNA, obtained with streptavidin-labeled GGGCGCG in M13mp18 digested with Bgl II; the arrow points to the streptavidin tag. Places where DNA strands cross each other (left side of height profile) are easily differentiated from labels. (b) Map of M13mp18 shows the location of GGGCGCG calculated from experimental data. Arrowheads indicate possible positions of the target sequence, based on the calculated distance from the restriction sites. Solid arcs indicate the correct paths, while incorrect paths are shown as dashed arcs (Woolley et al., 2000).

point by determining the haplotypes on a UDP-glucuronosyltransferase gene, UGT1A7 (Guillemette et al., 2000) whose enzyme product is involved in inactivation of carcinogens such as benzo[a]pyrene metabolites (Woolley et al., 2000). The UGT1A7 gene has two polymorphic sites (separated by $233 \mathrm{bp}$ ) that determine four alleles, each specifying different polypeptide chains (Figure 18a). Importantly, individuals who are heterozygous at both sites have a single genotype, but one of two haplotypes, $(* 1 / * 3)$ or $(* 2 / * 4)$, which cannot be differentiated using conventional methods. This ambiguity is crucial in screening, since each allele exhibits substantially different enzymatic activity towards targeted carcinogens. Significantly, the haplotypes of several subject samples have been unambiguously shown to be $(* 1 / * 3)$ by direct inspection of AFM images of labeled DNA (Figure 18b); that is, DNA molecules were only end-labeled with the streptavidin or the IRD800 probes.

We believe that direct haplotyping using SWNT AFM probes represents a significant advance over conventional approaches and could facilitate the use of SNPs for association and linkage studies of inherited diseases and genetic risk (Collins et al., 1997; Risch and Merikangas, 1996). To realize the greatest potential, will require that this methodology be extended to a high-throughput technique for genetic screening. The recent fabrication of multiple AFM tip arrays as large as $32 \times 32$ (Vettiger et al., 1999) could dramatically increase the number of samples typed in parallel, and the simplicity and distinctiveness of the AFM images of alternative haplotypes indicate that automated image analysis should be feasible. The implementation of these improvements could facilitate high-throughput haplotyping 


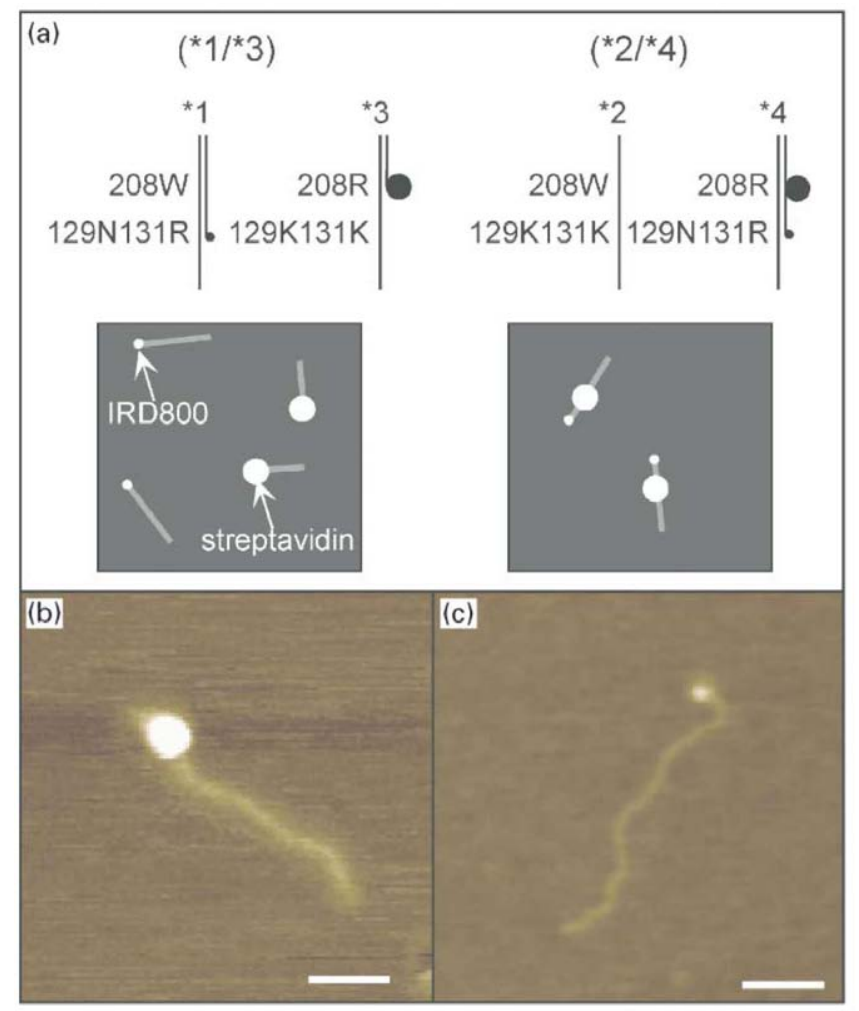

Figure 18. Direct haplotyping of UGT1A7 using SWNT probes: (a) Schematic showing haplotypes, alleles, genotypes, and locations of probes in samples analyzed. The $(* 1 / * 3)$ and $(* 2 / * 4)$ haplotypes, which have the same genotype (heterozygous at both loci), are specifically labeled at the 129N131R and 208R sites with IRD800 (small filled circle) and streptavidin (large filled circle), respectively. The double lines in the stick representations indicate duplex DNA, while single lines corresponded to single-stranded DNA. AFM images of a $(* 1 / * 3)$ sample should have an approximately equal number of fragments that are $\sim 210 \mathrm{~nm}$ long with IRD800 at one end, or $\sim 140 \mathrm{~nm}$ long with streptavidin at one end. In contrast, a (*2/*4) sample should contain $\sim 210 \mathrm{~nm}$ fragments with IRD800 at one end and streptavidin at $\sim 70 \mathrm{~nm}$. (b) Representative SWNT tip images of the $* 3$ allele (streptavidin end-labeled, $\sim 140 \mathrm{~nm}$ DNA) and the $* 1$ allele (IRD800 end-labeled, $\sim 210 \mathrm{~nm}$ DNA). The white bar corresponds to $50 \mathrm{~nm}$ in both images (Woolley et al., 2000).

using SWNT AFM probes. Lastly, the recent synthesis of carbon nanotubes with $0.25 \mathrm{~nm}$ radii (Sun et al., 2000), which are smaller than the spacing between DNA bases, indicates that further improvements in nanotube probes and labeling methods may even allow direct reading of the DNA sequence of fragments that are tens of kilobases in size.

\section{Functional imaging with nanotube probes}

The studies reviewed above have focused on the unique potential of carbon nanotube probes for reproducible, high-resolution structural imaging of proteins, protein assemblies, and protein-nucleic acid 
complexes. It is also possible to exploit the high normal force sensitivity of AFM for chemically and biologically sensitive measurements. In the past, the spatial resolutions of such measurements have been limited significantly by available probe tips. The well-defined chemistry possible at the ends of nanotube probes can overcome all previous limitations and thus opens up significant opportunities as described below.

\subsection{Chemical force microscopy and force spectroscopy}

In chemical force microscopy (CFM), the AFM tip is modified with specific chemical functional groups (Frisbie et al., 1994). This enables the tip to generate contrast dependent on the chemical properties of the sample from the friction signal in contact mode or the phase lag signal in tapping mode (Noy et al., 1998). Both of these signals are proportional to the adhesion between the tip and sample, and both can be measured simultaneously with topography. Functionalized tips have also been employed in force spectroscopy. In this mode of operation the tip is not scanned, but is brought into contact with a surface, then retracted. The forces applied to the tip during retraction are due to the interactions of tip and sample molecules. Force spectroscopy has been used to measure a variety of interactions including the intermolecular adhesion between fundamental chemical groups (Frisbie et al., 1994; Green et al., 1995; Noy and Noy; Vezenov et al., 1997), the unfolding of protein molecules (Rief et al., 1997), antigen-antibody interactions (Hinterdorfer et al., 1996), and DNA stretching and unbinding (Noy et al., 1997a).

Despite the progress made in chemically sensitive imaging and force spectroscopy using silicon and silicon nitride tips, these probes have very important limitations. First, the tips have a large radius of curvature making it difficult to control the number of active tip molecules and limiting significantly the lateral resolution. Second, the orientation and often the spatial location of the attached molecules cannot be controlled. This leads to uncertainty in the reaction coordinate for force spectroscopy, and increased non-specific interactions. Carbon nanotube tips can overcome these limitations. They have small radii of curvature for much higher resolution and can be specifically modified only at their very ends. Hence, functionalization of nanotube probes creates tips that have as few as one (depending on size) active molecular sites localized in a relatively controlled orientation. Modified SWNT tips could lead to subnanometer resolution in chemical contrast and binding site recognition.

\subsection{Nanotube tips in CFM and force spectroscopy}

Nanotube tips etched in air are expected to have carboxyl groups at their ends based on bulk studies of oxidized nanotubes (Hiura et al., 1995), although conventional analytical techniques have insufficient sensitivity to prove this point for isolated tubes. Chemical species present at the ends of nanotube tips can be studied with great sensitivity by measuring the adhesion of a nanotube tip on a chemically well-defined surface (Figure 19), for example, a hydroxyl $(-\mathrm{OH})$ terminated self-assembled monolayer (SAM). We have demonstrated the presence of carboxyl groups at the open ends of manually assembled MWNT and SWNT tips by measuring force titrations as shown in Figure 19b (Wong and Wong). In the force titration, the adhesion force between a nanotube tip and an SAM surface terminating in hydroxyl groups is recorded as a function of solution $\mathrm{pH}$, thus effectively titrating ionizable groups on the tip (Noy et al., 1997b; Vezenov et al., 1997). Significantly, force titrations recorded between pH 2 


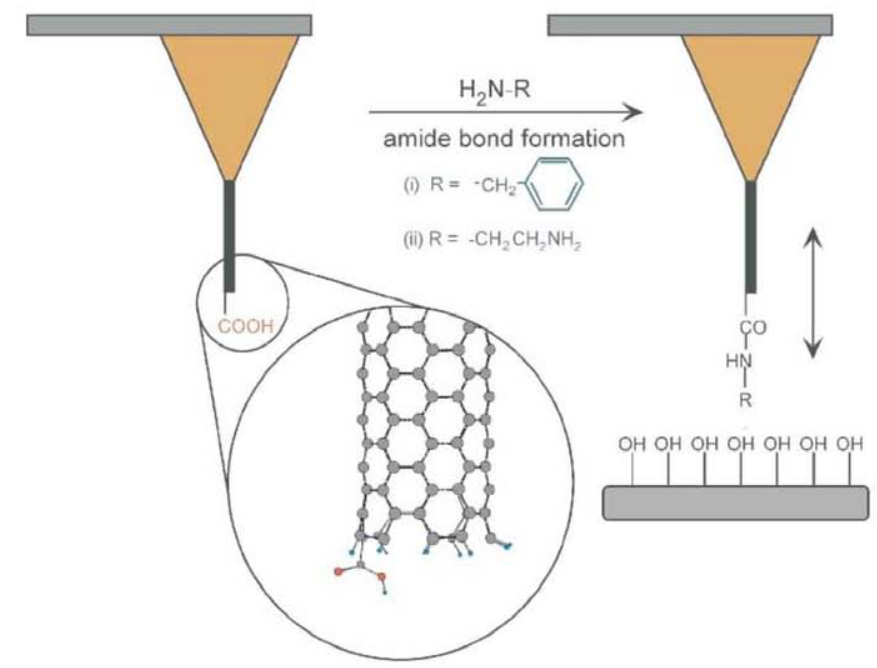

(a)

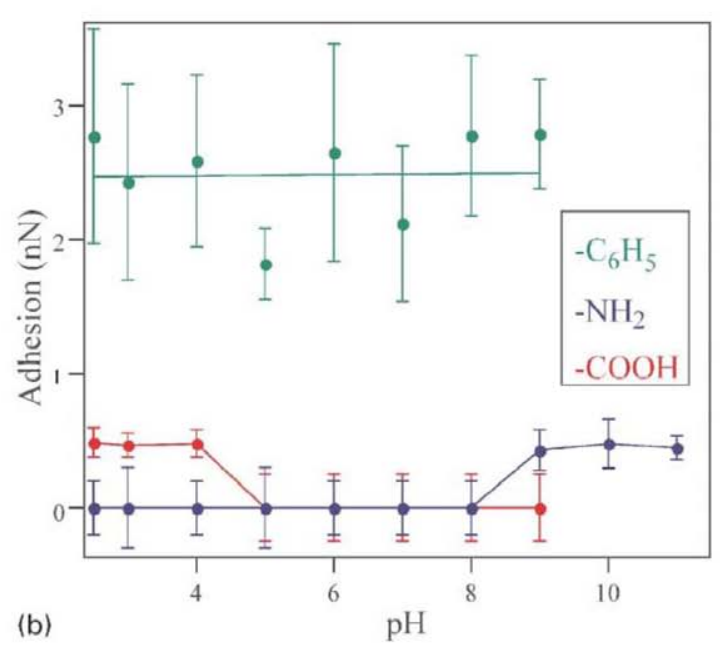

Figure 19. Covalent functionalization of carbon nanotube AFM tips: (a) Schematic illustrating the modification of the end of an SWNT, which terminates in a carboxylic acid functional group. The terminal carboxyl group is coupled with an amine, which bears the desired functionality, to form an amide bond. The tip end functionality is then assessed by force spectroscopy measurements on a chemically well-defined surface (Wong et al., 1998b). (b) Adhesion force as a function of $\mathrm{pH}$ (force titration) between assembled SWNT bundle tips and a hydroxyl-terminated SAM (11-thioundecanol on gold-coated mica). Each data point corresponds to the mean of 50-100 adhesion measurements, and the error bars represent one standard deviation (Wong et al., 1998c).

and 9 with MWNT and SWNT tips exhibit well-defined drops in the adhesion force at ca. $\mathrm{pH} 4.5$ that are characteristic of the deprotonation of a carboxylic acid. Moreover, the $\mathrm{pH}$ value where the adhesion drop occurs is similar to the $p K_{\mathrm{A}}$ of benzoic acid, thus suggesting that the carboxyl groups at the ends of nanotubes are solvent accessible.

The assembled SWNT and MWNT bundle tips also have been modified with organic and biological functionality by coupling organic amines to form amide bonds as outlined in Figure 19 (Wong and Wong). The success of this coupling chemistry was demonstrated by force titrations. Nanotube tips modified with benzylamine, which exposes nonionizable, hydrophobic functional groups at the tip end, yielded the expected $\mathrm{pH}$-independent interaction force on hydroxyl-terminated monolayers (Figure 19b). This covalent modification thus eliminates the prominent $\mathrm{pH}$-dependent behavior observed with the unfunctionalized tips. Moreover, force titrations with ethylenediamine-modified tips exhibit no adhesion at low $\mathrm{pH}$ and finite adhesion above $\mathrm{pH} 7$ (Figure 19b). These $\mathrm{pH}$-dependent interactions are consistent with our expectations for exposed basic amine functionality that is protonated and charged at low $\mathrm{pH}$ and neutral at high $\mathrm{pH}$.

Covalent reactions localized at nanotube tip ends represent a powerful strategy for modifying the functionality of the probe. However, the linking atoms that connect the tip and active group introduce 
conformational flexibility that may reduce the ultimate resolution. In an effort to develop a chemically sensitive probe without conformation flexibility, we have also explored the modification of the tips during the electrical etching process (Wong et al., 1999). The hypothesis behind this approach was that radical species, which can react with diatomic molecules such as $\mathrm{O}_{2}, \mathrm{H}_{2}$, and $\mathrm{N}_{2}$ are created during the electrical etching procedure used to optimize nanotube tip length. We tested this idea by carrying out the etching procedure in pure and mixed gas, and significantly, have demonstrated that these produce predictable changes in the chemical nature of the nanotube tip end. Specifically, force titrations carried out on tips modified in $\mathrm{O}_{2}, \mathrm{~N}_{2}$, and $\mathrm{H}_{2}$ exhibit behavior consistent with the incorporation of acidic, basic, and hydrophobic functionality, respectively, at the tip ends. We believe that these tips should enable chemical mapping at a resolution of a single SWNT tip, $0.5 \mathrm{~nm}$ or better.

In addition, these functionalized nanotube probes have been used to obtain chemically sensitive images of patterned monolayer and bilayer samples (Wong and Wong). Tapping mode images recorded with - $\mathrm{COOH}$ - and benzyl-terminated tips exhibit greater phase lag on the $-\mathrm{COOH}$ and $-\mathrm{CH}_{3}$ sample regions, respectively. In tapping mode AFM, we have shown that the differences in the tapping mode phase lag correlate directly with differences in intermolecular forces between the tip and sample: $\Delta \Phi_{\mathrm{o}}(k / Q) \propto \Delta W_{\mathrm{st}}$, where $\Delta \Phi_{\mathrm{o}}$ is the change in phase lag, $k$ is the spring constant, $Q$ is the cantilever quality factor, and $\Delta W_{\text {st }}$ is the difference between the work of adhesion for the tip interacting with two chemically distinct sample regions (Noy et al., 1998). Because the adhesion force between the carboxyl-terminated nanotube tip and the $\mathrm{COOH}$-terminated monolayer is greater than the $\mathrm{CH}_{3}$-terminated region, and the adhesion between the benzyl-terminated tip and the $\mathrm{CH}_{3}$-terminated region is greater than the $\mathrm{COOH}$-terminated region, these results were consistent with chemically sensitive imaging and our model for the driven oscillator described above.

The "chemical resolution" of functionalized manually assembled MWNT and SWNT tips has been tested on partial lipid bilayers (Wong et al., 1998c). Significantly, these studies have shown that an assembled SWNT tip could detect variations in chemical functionality with resolution down to $3 \mathrm{~nm}$, which is the same as the best structural resolution obtained with this type of tip. This resolution should improve with CVD SWNT tips, and recent studies bear this idea out (Cheung et al., 2000a). First, force titration data recorded on hydroxyl-terminated surfaces, which show finite adhesion below pH 5 and a repulsive interaction at higher $\mathrm{pH}$, show (as expected) that these tips terminate in a carboxyl group when shortened in air. The magnitudes of the adhesion forces also indicate that only 1-4 carboxyl groups are active in the measurements. Second, these CVD SWNT tips can be used for chemically sensitive mapping as shown in Figure 20. The high resolution of these tips is evident in the granular structure observed on samples prepared by standard lithography methods. This granularity corresponds to gold grain structure that typically cannot be resolved. We believe that these observations demonstrate that the CVD SWNT are sufficiently robust for solution work and that they have the potential for very high-resolution functional mapping.

In the future, we believe that these tips can be exploited in several ways for chemical or functional mapping in structural biology. For example, an important extension of our chemical mapping experiments is to protein surfaces, since the surface residues often define binding and orientation based on, for example, acidic or basic patches. Our structural studies of GroES (Cheung et al., 2000b), in which subunits were resolved with CVD SWNT tips (Figure 10), represent a good case in which to test this point. Examination of the crystal structure and electrostatic potential for GroES (Hunt et al., 1996) shows that 


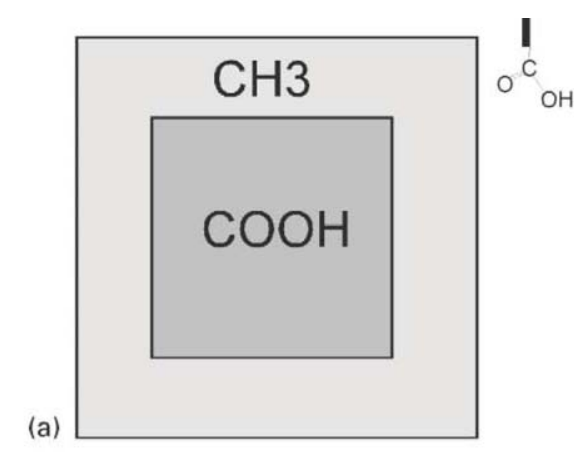

(b)
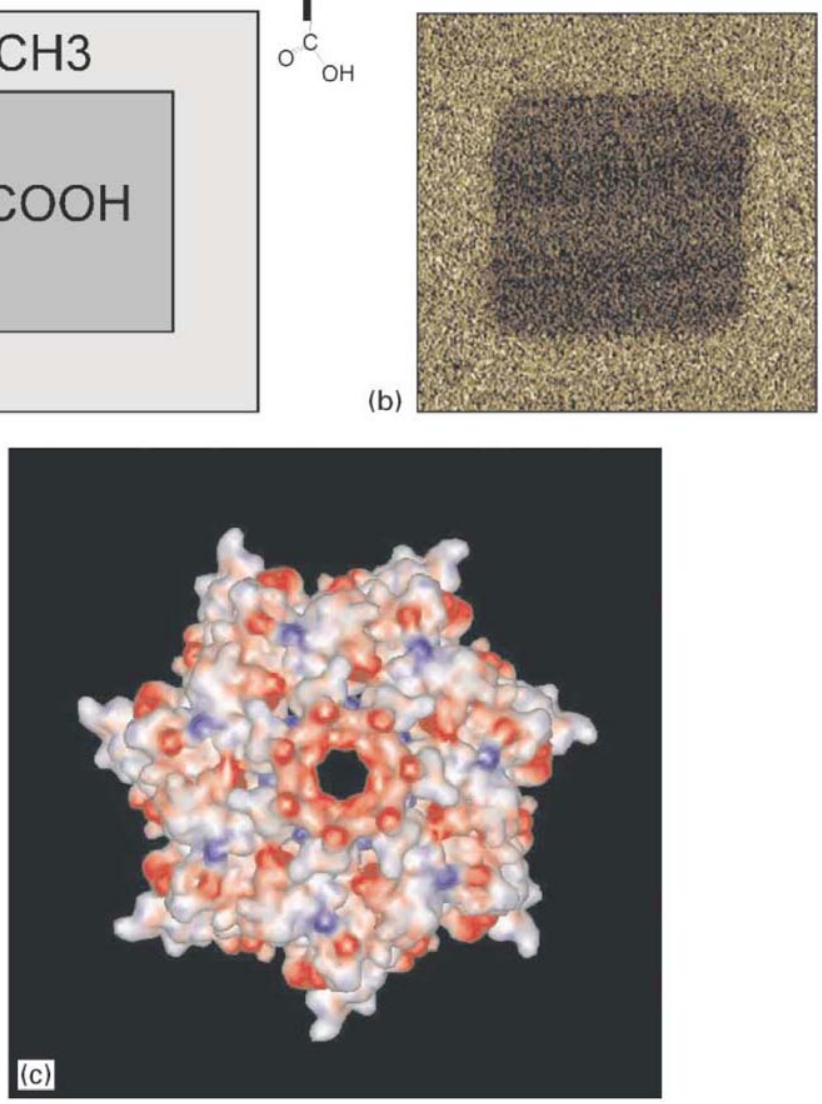

Figure 20. Chemical mapping with CVD SWNT probes: (a) Schematic illustrating a carboxyl-terminated CVD SWNT tip imaging a patterned SAM that terminates in methyl and carboxyl domains. (b) Tapping mode phase image recorded on an SAM surface patterned as in (a). The darker contrast in the middle square is consistent with the stronger $\mathrm{COOH}-\mathrm{COOH}$ vs. $\mathrm{COOH}-\mathrm{CH}_{3}$ interaction. (c) Plot of the electrostatic potential on the upper dome surface of GroES. Red corresponds to negative charge and blue to positive charge arising from acidic and basic residues, respectively.

there is a high density of acidic residues (14 glutamates; 2 each from each subunit) and accumulation of negative charge density at the top of dome (Figure 20c). Chemically sensitive mapping of the upper GroES surface using modified CVD SWNT tips, which are terminated with carboxyl or basic (obtained by coupling ethylenediammine or gas modification in $\mathrm{N}_{2}$ ) groups, should then show repulsive or attractive interactions, respectively, at the top of the dome.

In addition, modified nanotube probes could be used to study ligand-receptor binding/unbinding with control of orientation, and to map the position of ligand-receptor binding sites in proteins and 


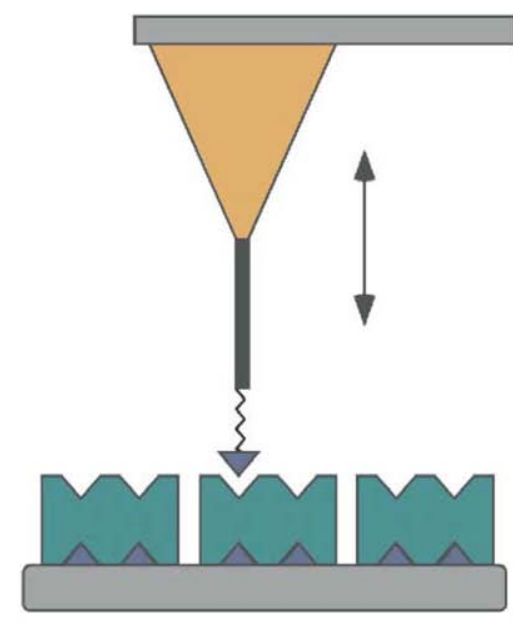

(a)

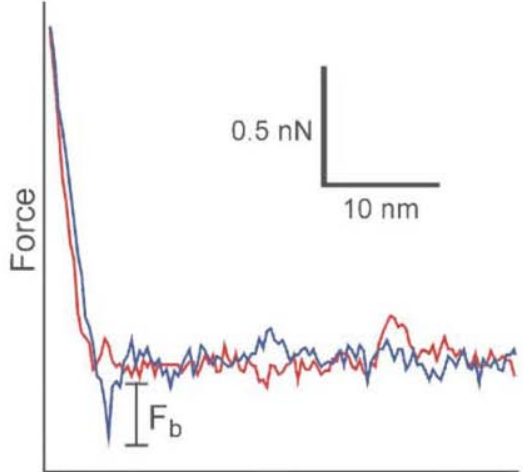

(b)

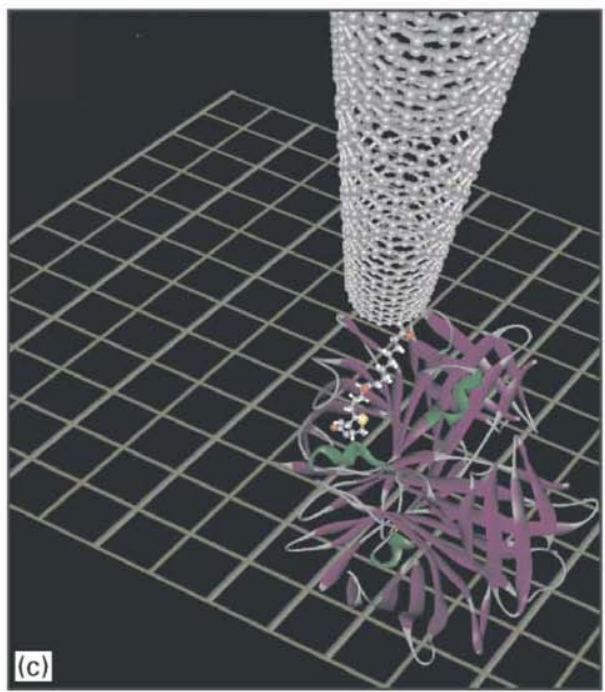

Figure 21. Nanotube AFM tips for single-molecule force spectroscopy and mapping: (a) Schematic illustrating the experiment, where the lower gray rectangle corresponds to a mica substrate, the green blocks correspond to streptavidin molecules, the dark blue inverted triangle is biotin covalently linked to a carbon nanotube (heavy black vertical line). (b) Representative force-displacement curve recorded with a biotin-modified MWNT tip in $\mathrm{pH} 7.0$ solution. The binding force, $200 \mathrm{pN}$, is consistent with unbinding of a single biotin ligand from streptavidin (Wong et al., 1998b). (c) Model of an SWNT tip (e.g., Figure 7) that has been modified with biotin and is interacting with a streptavidin molecule.

on cell surfaces with nanometer or better resolution. To illustrate this point, we have examined biotin-streptavidin, which is a model ligand-receptor system that has been widely studied (Livnah et al., 1993). 5-(biotinamido)pentylamine was covalently linked to nanotube tips via the formation of an amide bond, and then force-displacement measurements were recorded on mica surfaces containing immobilized streptavidin as shown in Figure 21 (Wong et al., 1998b). Force spectroscopy measurements show well-defined binding force quanta of ca. $200 \mathrm{pN}$ per biotin-steptavidin pair. Control experiments 
carried out with an excess of free biotin in solution, which blocks all receptor sites of the protein, and with unmodified nanotube tips showed no adhesion, and thus confirm that the observed binding force results from the interaction of nanotube-linked biotin with surface streptavidin. A key feature of these results compared to previous work (Florin et al., 1994; Lee et al., 1994), which relied on nonspecific attachment of biotin to lower resolution tips, is the demonstration that a single active ligand can be specifically localized at the end of a nanotube tip using well-defined covalent chemistry. With the current availability of individual SWNT tip via surface CVD growth, it is now possible to consider without extrapolation the mapping of ligand binding sites for a wide range of proteins (Figure 21c).

\section{Summary and future prospects}

AFM and related techniques show great promise as powerful tools for elucidating the structure and function of biological samples. The current limiting technology in AFM is the widely used, yet poorly defined $\mathrm{Si}$ or $\mathrm{Si}_{3} \mathrm{~N}_{4}$ tip. The studies of carbon nanotube AFM probe tips described in this review demonstrate that nanotubes overcome the critical limitations of $\mathrm{Si}$ and $\mathrm{Si}_{3} \mathrm{~N}_{4}$ tips, and may represent the ultimate AFM tip due to their cylindrical geometry, mechanical, and chemical properties. Our development of facile methods for the direct growth of nanotube tips by CVD allows the reproducible preparation of individual SWNT tips and represents a scalable approach that could make them widely available to the AFM and structural biology communities.

The availability of well-characterized individual SWNT tips will allow significant advances in AFM, as improvements in X-ray and electron sources have benefited X-ray diffraction and EM. Current progress on nanotube tips indicates that SWNT tips will allow sub-nanometer resolution on individual proteins in solution, and when the tip is functionalized provide similar resolution for mapping chemically distinct domains and ligand binding sites. We believe that the ability to obtain such functional information in vitro represents an important advantage compared to single particle cryoEM analysis, which has comparable structural resolution to SWNT probes. The range of new experiments that one can envision with SWNT probes, we believe, is truly unlimited, and likewise, the potential to contribute meaningful insight into the structure and function of complex protein and protein-nucleic acid systems is rich and exciting.

\section{Acknowledgements}

We thank Dr. J.D. Harper, Dr. P.T. Lansbury, Dr. G. Schnitzler and Dr. R.E. Kingston for valuable collaborations. C.M.L. acknowledges support of this work by the Air Force Office of Scientific Research and National Institutes of Health. J.H.H. acknowledges postdoctoral fellowship support from the National Institutes of Health.

\section{References}

Albrecht, T.R., Akamine, S., Carber, T.E. and Quate, C.F., 1989. J. Vac. Sci. Technol. A 8, pp. 3386-3396.

Arispe, N., Rojas, E. and Pollard, H.B., 1993. Proc. Natl. Acad. Sci. USA 90, pp. 567-571. 
Beauchamp, J.C. and Isaacs, N.W., 1999. Curr. Opin. Chem. Biol. 3, pp. 525-529.

Bergkvist, M., Carlsson, J., Karlsson, T. and Oscarsson, S., 1998. J. Colloid Interface Sci. 206, pp. 475-481.

Brookes, A.J., 1999. Gene 234, pp. 177-186.

Bustamante, C., Keller, D. and Yang, G., 1993. Curr. Opin. Struct. Biol. 3, pp. 363-372.

Bustamante, C. and Rivetti, C., 1996. Annu. Rev. Biophys. Biomol. Struct. 25, pp. 395-429.

Bustamante, C., Rivetti, C. and Keller, D.J., 1997. Curr. Opin. Struct. Biol. 7, pp. 709-716.

Cheung, C.-L., Chen, L., Lieber, C.M., 2000a. unpublished results.

Cheung, C.-L., Hafner, J.H. and Lieber, C.M., 2000. Proc. Natl. Acad. Sci. 97, pp. 3809-3813.

Cheung, C.-L., Hafner, J.H., Odom, T.W., Kim, K. and Lieber, C.M., 2000. Appl. Phys. Lett. 76, pp. 3136-3138.

Chiu, W., McGough, A., Sherman, M.B. and Schmid, M.F., 1999. Trends Cell Biol. 9, pp. 154-159.

Collins, F.S., Guyer, M.S. and Chakravarti, A., 1997. Science 278, pp. 1580-1581.

Cummings, B.J., Pike, C.J., Shankle, R. and Cotman, C.W., 1996. Neurobiol. Aging 17, pp. 921-933.

Dai, H., Hafner, J.H., Rinzler, A.G., Colbert, D.T. and Smalley, R.E., 1996. Nature 384, pp. 147-151.

Engel, A., Scheonenberger, C.-A. and Muller, D.J., 1997. Curr. Opin. Struct. Biol. 7, pp. 279-284.

Florin, E.-L., Moy, V.T. and Gaub, H.E., 1994. Science 264, pp. 415-417.

Frisbie, C.D., Rozsnyai, L.F., Noy, A., Wrighton, M.S. and Lieber, C.M., 1994. Science 265, pp. 2071-2074.

Fritz, J., Anselmetti, D., Jarchow, J. and Fernandez-Busquets, X., 1997. J. Struct. Biol. 119, pp. 165-171.

Fritz, M., Radmacher, M., Cleveland, J.P., Allersma, M.W., Stewart, R.J., Gieselmann, R., Janmey, P., Schmidt, C.F. and Hansma, P.K., 1995. Langmuir 11, pp. 3529-3535.

Fujihira, M. and Ohzono, T., 1999. Jpn. J. Appl. Phys. 38 Pt. 1, pp. 3918-3931.

Glaeser, R.M., 1999. J. Struct. Biol. 128, pp. 3-14.

Glusker, J.P., 1993. Bioanal. Instrum. 37, pp. 1-72.

Green, J.B.D., McDermott, M.T., Porter, M.D. and Siperko, L.M., 1995. J. Phys. Chem. 99, pp. 10960-10965.

Grigorieff, N., 1998. J. Mol. Biol. 277, pp. 1033-1046.

Guillemette, C., Ritter, J.K., Auyeung, D.J., Kessler, F.K., Housman, D.E., 2000. J. Biol. Chem., submitted for publication.

Guthold, M., Zhu, X., Rivetti, C., Yang, G., Thomson, N.H., Kasas, S., Hansma, H.G., Smith, H., Hansma, P.K. and Bustamante, C., 1999. Biophys. J. 77, pp. 2284-2294.

Hafner, J.H., Bronikowski, M.J., Azamian, B.R., Nikolaev, P., Rinzler, A.G., Colbert, D.T., Smith, K. and Smalley, R.E., 1998. Chem. Phys. Lett. 296, pp. 195-202.

Hafner, J.H., Cheung, C.-L. and Lieber, C.M., 1999. Nature 398, pp. 761-762.

Hafner, J.H., Cheung, C.-L. and Lieber, C.M., 1999. J. Am. Chem. Soc. 121, pp. 9750-9751.

Hafner, J.H., Cheung, C.-L., Lieber, C.M., 2000a. In preparation.

Hafner, J.H., Harper, J.D., Lansbury, P.T., Lieber, C.M., 2000b. J. Am. Chem. Soc., submitted for publication.

Hafner, J.H., Schnitzler, G., Cheung, C.L., Kingston, R.E., Lieber, C.M., 2000c. In preparation.

Han, W., Lindsay, S.M., Dlakic, M. and Harrington, R.E., 1997. Nature 386, p. 563.

Hansma, H.G., Laney, D.E., Bezanilla, M., Sinsheimer, R.L. and Hansma, P.K., 1995. Biophys. J. 68, pp. 1672-1677.

Harper, J.D., Wong, S.S., Lieber, C.M. and Lansbury, P.T., 1997. Chem. Biol. 4, pp. 119-125.

Harper, J.D., Wong, S.S., Lieber, C.M. and Lansbury, P.T., 1999. Biochemistry 38, pp. 8972-8980.

Hassig, C.A. and Schreiber, S.L., 1997. Curr. Opin. Chem. Biol. 1, pp. 300-308.

Henderson, R., 1995. Q. Rev. Biophys. 28, pp. 171-193.

Hinterdorfer, P., Baumgertner, W., Gruber, H.J., Schilcher, K. and Schindler, H., 1996. Proc. Natl. Acad. Sci. USA 93, pp. 3477-3481.

Hiura, H., Ebbesen, T.W. and Tanigaki, K., 1995. Adv. Mater. 7, pp. 275-276.

Hodge, S.E., Boehnke, M. and Spence, M.A., 1999. Nat. Genet. 21, pp. 360-361.

Hsia, A.Y., Masliah, E., McConLogue, L., Yu, G-Q., Tatsuno, G., Hu, K., Kholodenko, D., Malenka, R.C., Nicoll, R.A. and Mucke, L., 1999. Proc. Natl. Acad. Sci. USA 96, pp. 3228-3233.

Hunt, J.F., Weaver, A.J., Landry, S.J., Gierasch, L. and Deisenhofer, J., 1996. Nature 379, pp. 37-45.

Iijima, S., Brabec, C., Maiti, A. and Bernholc, J., 1996. J. Chem. Phys. 104, pp. 2089-2092.

Kadonaga, J.T., 1998. Cell 92, pp. 307-313.

Kasas, S., Thomson, N.H., Smith, B.L., Hansma, H.G., Zhu, X.X., Guthold, M., Bustamante, C., Kool, E.T., Kashlev, M. and Hansma, P.K., 1997. Biochemistry 36, pp. 461-468. 
Kim, S.H., 1998. Nat. Struct. Biol. 5, pp. 643-645.

Kornberg, R.D. and Lorch, Y., 1999. Cell 98, pp. 285-294.

Krishnan, A., Dujardin, E., Ebbesen, T.W., Yianilos, P.N. and Treacy, M.M.J., 1999. Phys. Rev. B 58, pp. 14013-14019.

Kuhlbrandt, W. and Williams, K.A., 1999. Curr. Opin. Chem. Biol. 3, pp. 537-543.

Lambert, M.P., Barlow, A.K., Chromy, B.A., Edwards, C., Freed, R., Liosatos, M., Morgan, T.E., Rozovsky, I., Trommer, B., Viola, K.L., Wals, P., Zhang, C., Finch, C.E., Krafft, G.A. and Klein, W.L., 1998. Proc. Natl. Acad. Sci. USA 95, pp. 6448-6453.

Lansbury, P.T., 1996. Acc. Chem. Res. 29, pp. 317-321.

Lee, G.U., Kidwell, D.A. and Colton, R.J., 1994. Langmuir 10, pp. 354-357.

Lemere, C.A., Bluzstzajn, J.K., Yamaguchi, H., Wisniewski, T., Saido, T.C. and Selkoe, D.J., 1996. Neurobiol. Dis. 3, pp. 16-32.

Li, J., Casell, A.M. and Dai, H., 1999. Surf. Interface Anal. 28, pp. 8-11.

Li, W.Z., Xie, S.S., Qian, L.X., Chang, B.H., Zou, B.S., Zhou, W.Y., Zhao, R.A. and Wang, G., 1996. Science 274, pp. $1701-1703$.

Lin, H. and Zhu, Y.J., 1999. Lal, R. Biochemistry 38, pp. 11189-11196.

Livnah, O., Bayer, E.A., Wilchek, M. and Sussman, J.L., 1993. Proc. Natl. Acad. Sci. USA 90, pp. 5076-5080.

Lorch, Y., Cairns, B.R., Zhang, M. and Kornberg, R.D., 1998. Cell 94, pp. 29-34.

Lu, J.P., 1997. Phys. Rev. Lett. 79, pp. 1297-1300.

Lyubchenko, Y.L. and Schlyakhtenko, L.S., 1997. Proc. Natl. Acad. Sci. USA 94, pp. 496-501.

Moechars, D., Dewachter, I., Lorent, K., Reverse, D., Baekelandt, V., Naidu, A., Tesseur, I., Spittaels, K., Van Den Haute, C., Checler, F., Godaux, E., Cordell, B. and Van Leuven, F., 1999. J. Biol. Chem. 274, pp. 6483-6492.

Mou, J., Czajkowsky, D.M., Sheng, S.J., Ho, R. and Shao, Z., 1996. FEBS Lett. 381, pp. 161-164.

Mou, J., Czajkowsky, D.M., Zhang, Y. and Shao, Z., 1995. FEBS Lett. 371, pp. 279-282.

Muller, D.J., Amrein, M. and Engel, A., 1997. J. Struct. Biol. 119, pp. 172-188.

Muller, D.J., Fotiadis, D. and Engel, A., 1998. FEBS Lett. 430, pp. 105-111.

Muller, D.J., Fotiadis, D., Scheuring, S., Muller, S. and Engel, A., 1999. Biophys. J. 76, pp. 1101-1111.

Muller, D.J., Sass, H.-J., Muller, S.A., Buldt, G. and Engel, A., 1999. J. Mol. Biol. 285, pp. 1903-1909.

Nikolaev, P., Bronikowski, M.J., Bradley, R.K., Rohmund, F., Colbert, D.T., Smith, K.A. and Smalley, R.E., 1999. Chem. Phys. Lett. 313, pp. 91-97.

Nishijima, H., Kamo, S., Akita, S. and Nakayama, Y., 1999. Appl. Phys. Lett. 74, pp. 4061-4063.

Noy, A., Frisbie, C.D., Rozsnyai, L.F., Wrighton, M.S. and Lieber, C.M., 1995. J. Am. Chem. Soc. 117, pp. 7943-7951.

Noy, A., Vezenov, D.V., Kayyem, J.F., Meade, T.J. and Lieber, C.M., 1997. Chem. Biol. 4, pp. 519-527.

Noy, A., Vezenoz, D.V. and Lieber, C.M., 1997. Annu. Rev. Mater. Sci. 27, pp. 381-421.

Noy, A., Sanders, C.H., Vezenov, D.V., Wong, S.S. and Lieber, C.M., 1998. Langmuir 14, pp. 1508-1511.

Orlova, E.V., Rahman, M.A., Gowen, B., Volynski, K.E., Ashton, A.C., Manser, C., van Heel, M. and Ushkaryov, Y.A., 2000. Nat. Struct. Biol. 7, pp. 48-53.

Pazin, M.J. and Kadonaga, J.T., 1997. Cell 88, pp. 737-740.

Perkins, S.J., Nealis, A.S., Sutton, B.J. and Feinstein, A., 1991. J. Mol. Biol. 221, pp. 1345-1366.

Rief, M., Gautel, M., Oesterhelt, F., Fernandez, J.M. and Gaub, H.E., 1997. Science 276, pp. 1109-1112.

Risch, N. and Merikangas, K., 1996. Science 273, pp. 1516-1517.

Scheuring, S., Ringler, P., Borgnia, M., Stahlberg, H., Muller, D.J., Agre, P. and Engel, A., 1999. EMBO J. 18, pp. $4981-4987$.

Schnitzler, G., Sif, S. and Kingston, R.E., 1998. Cell 94, pp. 17-27.

Selkoe, D.J., 1999. Nature 399, pp. A23-A31.

Shao, Z., Mou, J., Czajkowsky, D.M., Yang, J. and Yuan, J.-Y., 1996. Adv. Phys. 45, pp. 1-86.

Siegal, G., van Duynhoven, J. and Baldus, M., 1999. Curr. Opin. Chem. Biol. 3, pp. 530-536.

Sigler, P.B., Xu, Z., Rye, H.S., Burston, S.G., Fenton, W.A. and Horwich, A.L., 1998. Annu. Rev. Biochem. 67, pp. $581-608$.

Silverton, E.W., Navia, M.A. and Davies, D.R., 1977. Proc. Natl. Acad. Sci. USA 74, pp. 5140-5144.

Singh, S., Paola, T., Bustamante, C.J., Keller, D.J. and Capaldi, R., 1996. FEBS Lett. 397, pp. 30-34.

Sobel, E. and Lange, K., 1996. Am. J. Hum. Genet. 58, pp. 1323-1337.

Stowell, M.H., Miyazawa, A. and Unwin, N., 1998. Curr. Opin. Struct. Biol. 8, pp. 595-600.

Sun, L.F., Xie, S.S., Liu, W., Zhou, W.Y., Liu, Z.Q., Tang, D.S., Wang, G. and Qian, L.X., 2000. Nature 403, p. 384.)

Thess, A., Lee, R., Nikolaev, P., Dai, H.J., Petit, P., Robert, J., Xu, C.H., Lee, Y.H., Kim, S.G., Rinzler, A.G., Colbert, D.T., Scuseria, G.E., Tomanek, D., Fischer, J.E. and Smalley, R.E., 1996. Science 273, pp. 483-487. 
Treacy, M.M.J., Ebbesen, T.W. and Gibson, J.M., 1996. Nature 381, pp. 678-680.

Vesenka, J., Manne, S., Giberson, R., Marsh, T. and Henderson, E., 1993. Biophys. J. 65, pp. 992-997.

Vettiger, P., Brugger, J., Despont, M., Drechsler, U., Durig, U., Haberle, W., Lutwyche, M., Rothuizen, H., Stutz, R., Widmer, R. and Binnig, G., 1999. Microelectron. Engng 46, pp. 11-17.

Vezenov, D.V., Noy, A., Rozsnyai, L.F. and Lieber, C.M., 1997. J. Am. Chem. Soc. 119, pp. 2006-2015.

Viani, M.B., Schaffer, T.E., Chand, A., Rief, M., Gaub, H.E. and Hansma, P.K., 1999. J. Appl. Phys. 86, pp. $2258-2262$.

Viani, M.B., Schaffer, T.E., Paloczi, G.T., Pietrasanta, L.I., Smith, B.L., Thompson, J.B., Richter, M., Rief, M., Gaub, H.E., Cleland, A.N., Hansma, H.G. and Hansma, P.K., 1999. Rev. Sci. Instrum. 70, pp. 4300-4303.

Walsh, D.M., Lomakin, A., Benedek, G.B., Condron, M.M. and Teplow, D.B., 1997. J. Biol. Chem. 272, pp. $22364-22372$.

Wong, E.W., Sheehan, P.E. and Lieber, C.M., 1997. Science 277, pp. 1971-1975.

Wong, S.S., Harper, J.D., Lansbury, P.T. and Lieber, C.M., 1998. J. Am. Chem. Soc. 120, pp. 603-604.

Wong, S.S., Joselevich, E., Woolley, A.T., Cheung, C.-L. and Lieber, C.M., 1998. Nature 394, pp. 52-55.

Wong, S.S., Woolley, A.T., Joselevich, E., Cheung, C.-L. and Lieber, C.M., 1998. J. Am. Chem. Soc. 120, pp. 8557-8558.

Wong, S.S., Wooley, A.T., Joselevich, E. and Lieber, C.M., 1999. Chem. Phys. Lett. 306, pp. 219-225.

Wong, S.S., Woolley, A.T., Odom, T.W., Huang, J.-L., Kim, P., Vezenov, D.V. and Lieber, C.M., 1998. Appl. Phys. Lett. 73, pp. $3465-3467$.

Woolley, A.T., Guillemette, C., Cheung, C.-L., Housman, D.E. and Lieber, C.M., 2000. Nat. Biotech. 18, pp. 760-763.

Wuthrich, K., 1995. Acta Crystallogr. D 51, pp. 249-270.

Wuthrich, K., 2000. Nat. Struct. Biol. 7, pp. 188-189.

Zhang, Y., Sheng, S.J. and Shao, Z., 1996. Biophys. J. 71, pp. 2168-2176.

Zlatanova, J., Leuba, S.H. and van Holde, K., 1998. Biophys. J. 74, pp. 2554-2566. 